\title{
§ IV Additional texts from Nineveh
}

Some fragments might belong to the Nineveh UGU or IGI, but they cannot as yet be properly placed. Other texts, not belonging to the IGI or the UGU Treatises, comprise loose collections of material that have not been edited into a standardized treatise. Such texts might be ahû 'extraneous / non-canonical' collections or oral collections, recorded by scribes in the Royal Library of Ashurbanipal (Panayotov 2018: 112 and elsewhere in BAM 9).

The two-column fragmentary tablet NF (BAM 520) probably represents the fourth tablet of the Nineveh IGI treatise. The main clue that a fourth tablet existed in Nineveh comes from the Assur Medical Catalogue (Panayotov 2018: 97, edition in BAM 9: 210):

\section{EYES}

8) $\mathrm{B} 3{ }^{\prime}(+) \mathrm{A} 8$

DIŠ NA IGI.MIN-šú 'GIG :? D[IŠ NA IGI.MIN-šú mur-din- $n] i$ `DIRI? 'If a man's eyes are sick. [If a man's eyes] are full of [(a)murdin]nu ('bramble').'

9) $\mathrm{B} 4{ }^{\prime}(+) \mathrm{A} 9$

DIŠ NA IGI.MIN-šú LÙ.LÙ [: DIŠ NA? x x x x] 'x x x-šú ?!? 'DIRI' 'If a man's eyes are constantly troubled. [If a man's] ... are full of [...]. ‘

10) $\mathrm{B} 5$ ’ (+)A10

NÍGIN 4 DUB.MEŠ DIŠ NA IGI.MIN-šú G[IG EN (...) šum $\left.{ }_{4}^{?}-m\right] a^{\text {? }}$ ${ }^{r} \mathrm{IGI}_{\text {.SIG }} . \mathrm{SIG}_{7}{ }^{\urcorner} u$ sin-' $l u-u r-m a-a^{\urcorner}$'Total of four tablets (of the section) 'If a man's eyes are si[ck.' Including (prescriptions) for the case] that (he has) jaundice or night-blindness.'

Another argument in favour of NF (BAM 520) as the fourth tablet of IGI is the fact that it shares recipes with IGI 3, since a common feature of the Nineveh Medical Encyclopaedia is that the same prescription could be used in different contexts, as happens in the first three tablets of the IGI-Treatise.

\section{§ IV.1 NF (BAM 520)}

Base Manuscript: NF (BAM 520 (+) AMT 14/2), collated.

Museum number: $\quad$ British Museum, K 8784 + K $9503+81-7-27,85$ (+) K 11723. Join K $8784+$ is courtesy of Eric Schmidtchen.

Origin and date: Nineveh, 7 th century BC.

Type of tablet: Middle fragment of a two column, portrait-oriented tablet. The vertical dividing rulings were made by a twisted thread. There are no holes. 
Measurements: ca. $10 \times 14 \times 3,5 \mathrm{~cm}$.

Copy: $\quad$ BAM 520, AMT 14/2.

Photo/CDLI nr.: $\quad$ Plates 31-32a/P398154/P397754.

Literature: $\quad$ Caplice 1967: text 33; Finkel 1976: 254; Köcher 1980b: xiv (BAM 520); Maul 1994: 461ff.; Fincke 2000: 331; Scurlock and Andersen 2005: 793; Scurlock 2006: 782; Scurlock 2014: 756; Attia 2015: 71 and 79; Scurlock 2017: 286; Zomer 2018.

\section{§ IV.1.1 Related Manuscripts containing IGI 3 from Nineveh}

The text included represent parallels and partial parallels from the other tablets of the IGI treatise.

Manuscript: $\quad$ NE (BAM 516), see $\$$ III.1, parallels: 8ff.’.

\section{§ IV.1.2 Related other Manuscripts from Nineveh}

These texts represent parallels and partial parallels which are considered important for the compilation of the IGI-treatise. They come from other tablets and fragments from Nineveh containing prescriptions for sick eyes or from the UGU-treatise.

Manuscript: $\quad$ NL (AMT 49/4) collated, parallels: 26’.

Museum number: $\quad$ British Museum, K $2462+\mathrm{K} 7824$.

Origin and date: Nineveh, 7th century BC.

Type of tablet: $\quad$ Fragment from the top left of a two-column, portrait-oriented tablet. The vertical orienting rulings (see edge of col. iv) were made by a twisted thread. There are no round holes. This fragment is part of the $3^{\text {rd }}$ tablet of the Nineveh Treatise 'BRONCHIA', DIŠ NA KIR 4 -šu DUGUD.

Measurements: ca. $6,2 \times 6,5 \times 2,2 \mathrm{~cm}$.

Copy: AMT $49 / 4$.

CDLI nr.: P394449.

Literature:

Scurlock and Andersen 2005: 781; Scurlock 2014: 298f., 752; Panayotov 2018: 101; Bácskay 2018a: 93f. and 234ff.

Manuscript: $\quad$ NM (BAM $489+508)$, collated, parallels: 29ff.'.

Museum number: $\quad$ British Museum, K 239 + K 2509 + K 3261 + K 9080.

Origin and date: Nineveh, 7th century BC. 
Type of tablet: $\quad$ Fragmentary two-column, portrait-oriented tablet. The vertical orienting rulings were done by an instrument or stylus. There are no round holes.

Measurements: $\quad$ ca. 19,3 $\times 12,4 \times 2,7 \mathrm{~cm}$.

Copy:

Finkel 1976: pl. 20; BAM $489+508$.

Photo/CDLI nr.: $\quad$ Plates 44/P393804.

Literature: $\quad$ Finkel 1976: nr. 25, ms. L, pl. 20; Köcher 1980a: xxviii (BAM 489), xxxv-xxxvi (BAM 508); Scurlock and Andersen 2005: 793; Scurlock 2006: 782; Attia and Buisson 2007: 48; Scurlock 2014: 756; Scurlock 2017: 286, 288; Zomer 2018.

Manuscript: $\quad$ NN (K 6329), collated, parallels: 29ff.'.

Museum number: $\quad$ British Museum, K 6329.

Origin and date: Nineveh, 7th century BC.

Type of tablet: Middle fragment of three'-column, portrait-oriented tablet. The vertical orienting rulings were done by an instrument or stylus. There are no round holes.

Measurements: $\quad$ ca. $8,1 \times 5,3 \times \ldots \mathrm{cm}$.

Photo/CDLI nr.: $\quad$ Plate 45; P396459.

Literature: $\quad$ Köcher 1964: xv (BAM 216); Finkel 1976: 57, 253f., 279; Köcher 1980a: xxxvi (BAM 508); Köcher 1980b: xiv (BAM 520); Scurlock

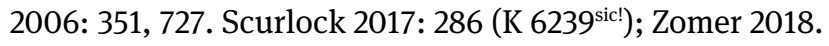

Manuscript: $\quad$ NO (K 8211+... (+) BM 98589+ ...), collated, parallels: 29ff.'.

Museum number: $\quad$ British Museum, K 8211 + K 4609a (+) BM 98589 + 98584 (K 5416a).

Origin and date: Nineveh, 7th century BC.

Type of tablet: $\quad$ Fragments of three-column, portrait-oriented tablet. The vertical orienting rulings were done by an instrument or stylus. There are no round holes.

Measurements: $\quad$ BM 98584 + BM 98589: ca. 10,6 $\times 13,1 \times 2,6-3,5$ (towards the middle) cm.; ca. K $8211+$ K 4609a: 13,4×13,8 2,6 - 3,6 (towards the middle) cm.; K 8211 + K 4609a (+) BM 98584 + BM 98589: ca. 13,4×23,6+×2,6 - 3,6 (towards the middle) $\mathrm{cm}$.

Copy: $\quad$ ABRT 2/11; AMT 45/5; Finkel 1976: pl. 27.

Photo/CDLI nr.: $\quad$ Plate 45-46/P397537+P395655+P396019.

Literature: $\quad$ Köcher 1964: Xv (BAM 216); Finkel 1976: 77, 146, 252, no. 30, ms. S, pl. 27; Köcher 1980a: xxxvi (BAM 508); Köcher 1980b: xiv (BAM 520); Scurlock 2006: 351, 727; Böck 2007: 328 (diverse); Scurlock 2017: 286; Zomer 2018.

Manuscript: $\quad$ NT (AMT 85/2), see § IV.7, parallels: 13’, 15’. 
Manuscript: $\quad$ NX (BAM 482), see $§$ V.2, parallels: 29’.

Manuscript: $\quad$ NXa (AMT 19/1, AMT 20/1), collated, parallels: 29’.

Museum number: British Museum, K 6066 + Sm 1063

Origin and date: Nineveh, 7th century BC.

Type of tablet: $\quad$ Middle fragment of a two-column, portrait-oriented tablet. The vertical dividing rulings were made by a twisted thread. There are numerous round holes pierced in patterns at empty spaces. Serialized tablet belonging to UGU 2 duplicating NX (BAM 482).

Measurements: $\quad$ ca. $11,2 \times 17,1 \times 2,3-3,1$ (towards the middle) $\mathrm{cm}$.

Copy: $\quad$ AMT 19/1, AMT 20/1.

CDLI nr.: $\quad$ P396343.

Literature: $\quad$ Edition and secondary literature in Attia and Buisson 2003.

\section{§ IV.1.3 Related Manuscripts from Different Cities}

The texts included here represent parallels and partial parallels which are considered important for the compilation of the IGI-treatise. These are manuscripts coming from other periods and from cities other than Nineveh.

\section{Sigla notations:}

A = Assur, Late Assyrian. A, B, C, ... designate the different manuscripts.

$\mathbf{d k}=$ Dūr Kurigalzu, Middle Babylonian.

$\mathbf{X}=$ unknown provenance, Late Assyrian. A, B, C, ... designate the different manuscripts.

$\mathbf{s}=$ Sippar, Late Babylonian. A, B, C, ... designate the different manuscripts.

$\mathbf{u}=$ Uruk, Late Babylonian. A, B, C, ... designate the different manuscripts.

Initial capital letters designate tablets in Assyrian script or northern scripts, and small letters tablets in Babylonian script.

Parenthesis [0] on a siglum designate a parallel text and not a duplicate.

\section{Assyrian}

Manuscript:

AI (LKA 145), not collated, parallels: 29ff.'.

Museum number:

Istanbul?

Origin and date: Assur, $8^{\text {th }}$ or $7^{\text {th }}$ century BC.

Type of tablet: Landscape-oriented tablet.

Copy: LKA 145.

CDLI nr.: CDLI P414054.

Literature: Scurlock 2017: 286; Zomer 2018. 
Manuscript: $\quad$ AJ (BAM 216), collated, parallels: 29'.

Museum number: Vorderasiatisches Museum, Berlin, VAT 8911.

Origin and date: Assur, $8^{\text {th }}$ or $7^{\text {th }}$ century BC.

Type of tablet: One-column tablet with round holes at empty spaces.

Measurements: $\quad$ ca. $17,9 \times 9,3 \times 2,3$ (towards the middle) $\mathrm{cm}$.

Copy:

BAM 216.

CDLI nr.:

CDLI P285303.

Literature:

Köcher 1964: viv-xv (BAM 216); Scurlock 2006: nos. 87, 113, 114b, 117, 137c, 182, 184, 205, 206, 208, 211, 212, 214, 248, 249, 295. Scurlock 2017: 286.

Manuscript:

AK (AO 11447), collated, parallels: 29'.

Museum number:

Louvre, AO 11447.

Origin and date:

Assur, $8^{\text {th }}$ or $7^{\text {th }}$ century BC.

Type of tablet:

One-column tablet with round holes at empty spaces.

Measurements:

ca. $23 \times 10,7 \times 2 \mathrm{~cm}$.

Copy:

JMC 10: $5 f$.

CDLI nr.:

P493334.

Literature:

Edition and secondary literature in Geller 2007b; Scurlock 2017: 285ff.

Manuscript:

XA (BM 123362), collated, parallels:29ff.'.

Museum number:

British Museum, BM 123362.

Origin and date: Unclear, 7th century BC.

Type of tablet:

Rests of the lower right hand corner of a two-column tablet. The tablet is unearthed in Niniveh but it is unclear from where the tablet originates. The script might suggest Assur as well. There are round holes in empty spaces.

Measurements: $\quad$ ca. $8,4 \times 8,7 \times 2,5-3,0$ (towards the middle) $\mathrm{cm}$.

Copy:

Plate 47.

Photo/CDLI nr.: $\quad$ Plate 47-48/P422463.

Literature:

Lambert and Millard 1968: 19; Scurlock 2006: 351, 727; Scurlock 2017: 286; Zomer 2018.

\section{Babylonian}

Manuscript: $\quad$ dk (Sumer 9/29), not collated, parallels: 29ff.'.

Museum number: National Museum of Iraq, Baghdad, IM 49981

Origin and date: Dūr Kurigalzu, Middle Babylonian.

Type of tablet: Fragment of a one-column, portrait oriented tablet?

Copy:

Sumer 9: no. 29. 
Literature: $\quad$ Gurney 1953: no. 29. Finkel 1976: 254, ms. i; Zomer 2018.

Manuscript: $\quad$ SA (IRAQ 65), see $§$ III.4, parallels: 29’.

Manuscript: $\quad$ uC (SpTU 22+85), not collated, parallels: 13f.'.

Museum number: $\quad$ National Museum of Iraq, Baghdad, Excavation no. W 22664.

Origin and date: Uruk, Late Babylonian.

Type of tablet: One-column, portrait oriented tablet.

Measurements: $\quad 6,5 \times 5,1 \mathrm{~cm}$.

Copy: $\quad$ SpTU $22+85$.

CDLI nr.: $\quad$ P348627+ P348689.

Literature: $\quad$ von Weiher 1983: no. 22; von Weiher 1988: no. 85; Fincke 1998;

Fincke 2000: 241 fn. 1792; Scurlock and Andersen 2005: 187.

\section{Edition of NF (BAM 520)}

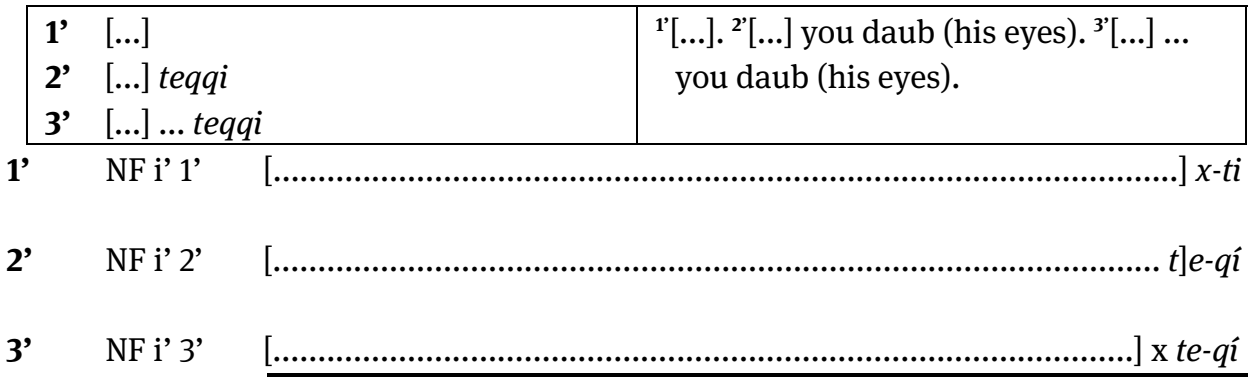

\begin{tabular}{|c|c|c|}
\hline $\begin{array}{l}4 \\
5 \\
6 \\
6 \\
7\end{array}$ & $\begin{array}{l}{[\ldots] \text {... }} \\
{[. . .] \text { ina šamni tasâk ašar šanîmma }} \\
{[\ldots . .] \text { ašar šanimma }} \\
\text { [...] innišsu teqqi }\end{array}$ & $\begin{array}{l}{ }^{4}\left[\ldots . . . .^{5}[\ldots] \text { you pound [in] oil, ditto. }{ }^{6}[\ldots]\right. \\
\text { ditto }\left(=\text { you daub his eyes). }{ }^{7}[\text {,... you }\right. \\
\text { daub his eyes. }\end{array}$ \\
\hline 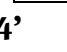 & NF i' 4' & ......] TE GI \\
\hline & NF i' 5 , & (I) SÚD 'KIMIN' \\
\hline & NF i' 6 ' & KIMIN \\
\hline & 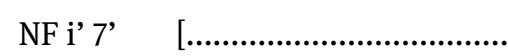 & 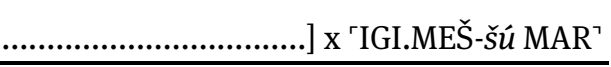 \\
\hline
\end{tabular}

Overview of Manuscripts and Parallels concerning NF (BAM 520): AI=LKA 145; AJ=BAM 216; AK=AO 11447; dk=Sumer 9/29; NE=BAM 516; $\mathbf{N F}=$ BAM 520 (+); NL=AMT 49/4; NM=BAM 489+508; NN=K 6329; NO=K 8211+... (+) BM 98589...; NT=AMT 85/2; NX=BAM 482; NXa=AMT 19/1; SA=IRAQ 65; uC=SpTU22+; XA=BM 123362 


\begin{tabular}{|c|c|c|c|}
\hline $\begin{array}{l}8^{\prime} \\
9^{\prime}\end{array}$ & $\begin{array}{l}\text { [kīma kan } \\
\text { tasâkma] } \\
\text { teqqīma i }\end{array}$ & $\begin{array}{l}\text { müșa zalāqa } \mathrm{x} \\
\text { māmta }[\ldots]\end{array}$ & 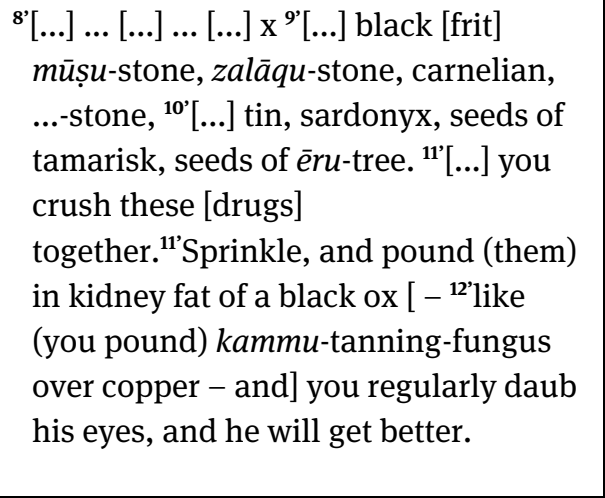 \\
\hline & $\begin{array}{l}\text { NF i' 8' } \\
(\mathrm{NE}) \text { i } 3\end{array}$ & \multicolumn{2}{|c|}{ 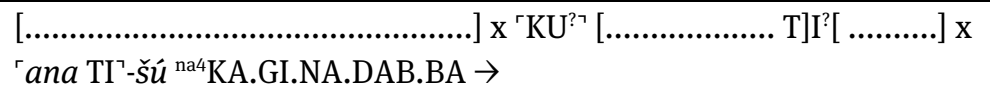 } \\
\hline & $\begin{array}{l}\text { NF i' 9' } \\
\text { (NE) i 3f. }\end{array}$ & \multicolumn{2}{|c|}{ 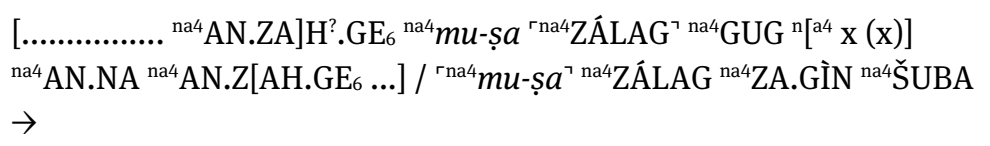 } \\
\hline $0^{\prime}$ & $\begin{array}{l}\text { NF i' 10' } \\
\text { (NE) i } 4 f .\end{array}$ & \multicolumn{2}{|c|}{ 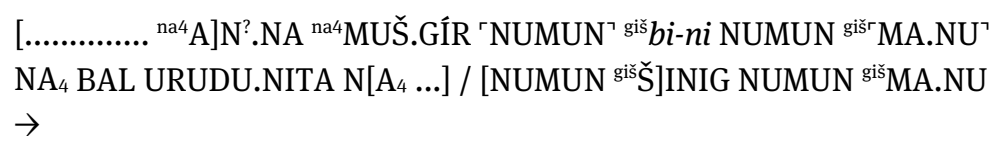 } \\
\hline & $\begin{array}{l}\text { NF i' } 11 \\
(\mathrm{NE}) \text { i } 5 \\
(\mathrm{NE}) \text { i } 6\end{array}$ & \multicolumn{2}{|c|}{$\begin{array}{l}\text { [......... Ú.M]EŠ ŠEŠ 1-niš GAZ ta-ša-pah ina İ.UDU ÉLLAG GU } \mathrm{G}_{4} \mathrm{GE}_{6} \\
\text { NUMUN áš-li NITA }{ }^{\text {na4}} a \hat{s}-h a[r \text {...] } \\
\text { [ta-š] } a-p a h \text { ina İ.UDU ÉLLAG GU } \mathrm{GE}_{6} \rightarrow\end{array}$} \\
\hline
\end{tabular}

12' NF i' 12' IG]I.MEŠ-šú ka-a-a-man-nam-ma MAR-ma ina-eš (NE) i 6 $\mathrm{GIN}_{7}$ kám-ma ina UGU URUDU SÚD- $m[a$...]

13’ [šumma amēlu enūma birṣa] ìtanammaru šalāšššu kīam liqbi ša Enlil u Ninlil

14' [anāku ša Ištar u Nanāya?] anāku iqabbīma iballuṭ
${ }^{13}$ 'If a man] constantly [sees a flash of light:] he should say three times as follows: ' ${ }^{14} \mathrm{I}$ belong to ${ }^{13 ’}$ Enlil and Ninlil, ${ }^{14}$ [I belong to Ištar and Nanaya?].' He says this, then he should recover.

Overview of Manuscripts and Parallels concerning NF (BAM 520): AI=LKA 145; AJ=BAM 216; AK=AO 11447; dk=Sumer 9/29; NE=BAM 516; $\mathbf{N F}=$ BAM 520 (+); NL=AMT 49/4; NM=BAM 489+508; NN=K 6329; NO=K 8211+... (+) BM 98589...; NT=AMT 85/2; NX=BAM 482; NXa=AMT 19/1; SA=IRAQ 65; uC=SpTU22+; XA=BM 123362 
13' NF i' 13’ [DIŠ NA e-nu-ma bir-ș] $a^{\ulcorner}$IGI-ru? 3-šú ki-a-am liq-bi šâ ${ }^{\mathrm{d}+}$ En-líl u ${ }^{\mathrm{d}}$ Nin-líl (uC) i 18f. DIŠ NA e-nu-ma bir-și i-ta-nam-ma-ru ki-a-am DUG4.GA / šáa ${ }^{\mathrm{d}+} E n-l i ́ l u$ ${ }^{\mathrm{d} N i n-l i ́ l}$

(NT) 4’ DIŠ NA ina IGI.MEŠ-šú bir-și I[GI(.IGI)-mar

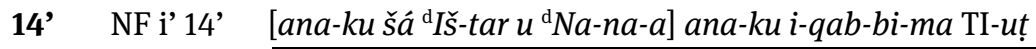

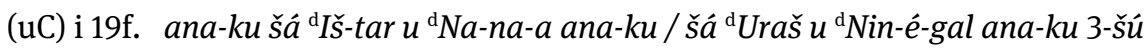
DUG4.GA-ma DIN

\begin{tabular}{|c|c|}
\hline $\begin{array}{l}\text { 15’ }[\text { šumma ... ana imitti] izzizzu teleqqe } \\
\text { ina išāti tukabbab } \\
\text { 16' }[\text {... ina] dišpi himēti tasâk ìnīšu } \\
\text { teqqīma ina'eš }\end{array}$ & $\begin{array}{l}{ }^{15}[\text { If ... ] you take [...] which stands [on } \\
\text { the right] and you burn (it) in the fire. } \\
{ }^{16}[\ldots . .] \text { You pound (it) [in] honey and } \\
\text { ghee. You daub his eyes and then he } \\
\text { should get better. }\end{array}$ \\
\hline $\begin{array}{l}\text { NF i' } 15 \\
\text { (NT) 6’ }\end{array}$ & 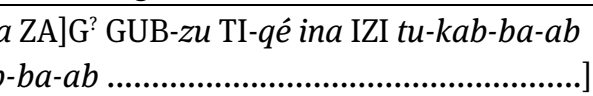 \\
\hline
\end{tabular}

16' NF i' 16' ina L]ÀL İ.NUN SÚD IGI.MIN-šú te-eq-qí-ma ina-eš

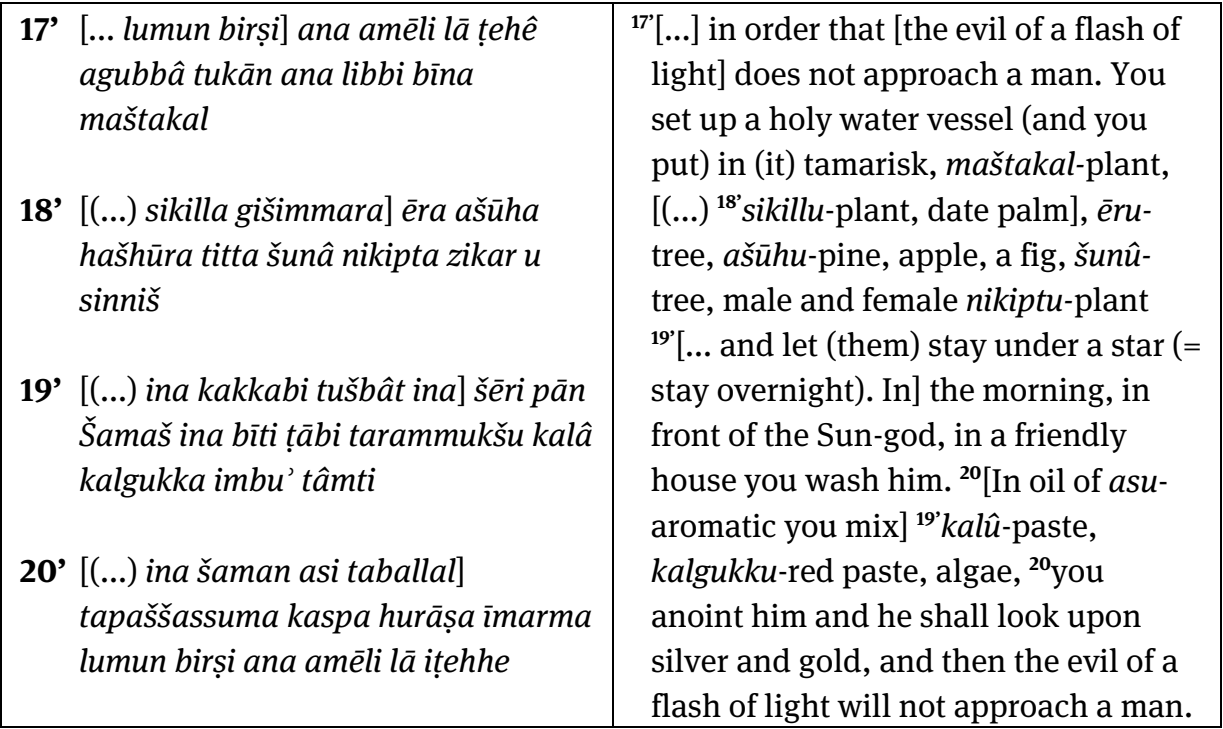

Overview of Manuscripts and Parallels concerning NF (BAM 520): AI=LKA 145; AJ=BAM 216; AK=AO 11447; dk=Sumer 9/29; NE=BAM 516; $\mathbf{N F}=$ BAM 520 (+); NL=AMT 49/4; NM=BAM 489+508; NN=K 6329; NO=K 8211+... (+) BM 98589...; NT=AMT 85/2; NX=BAM 482; NXa=AMT 19/1; sA=IRAQ 65; $\mathbf{u C}=$ SpTU22+; XA=BM 123362 
17’ NF i’ 17’ [... HUL bir-ṣ] $i^{?}$ `a-na LÚ NU TE-e A.GÚB.BA GIN-an ana ŠÀ-bi gišŠINIG ú $\mathrm{IN}_{6}$.ÚŠ

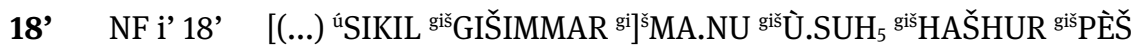

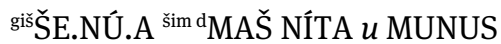

19’ NF i’ 19' [(...) ina UL tuš-bat ina Á.G]Ú.ZI.GA IGI dUTU ina É ța-bi TU ${ }^{2}$-šú ${ }^{\mathrm{im}} \mathrm{KAL}$ imKAL.GUG KA.A.AB.BA

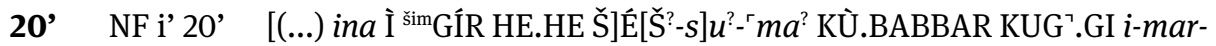
ma HUL bir-și ana LÚ NU TE

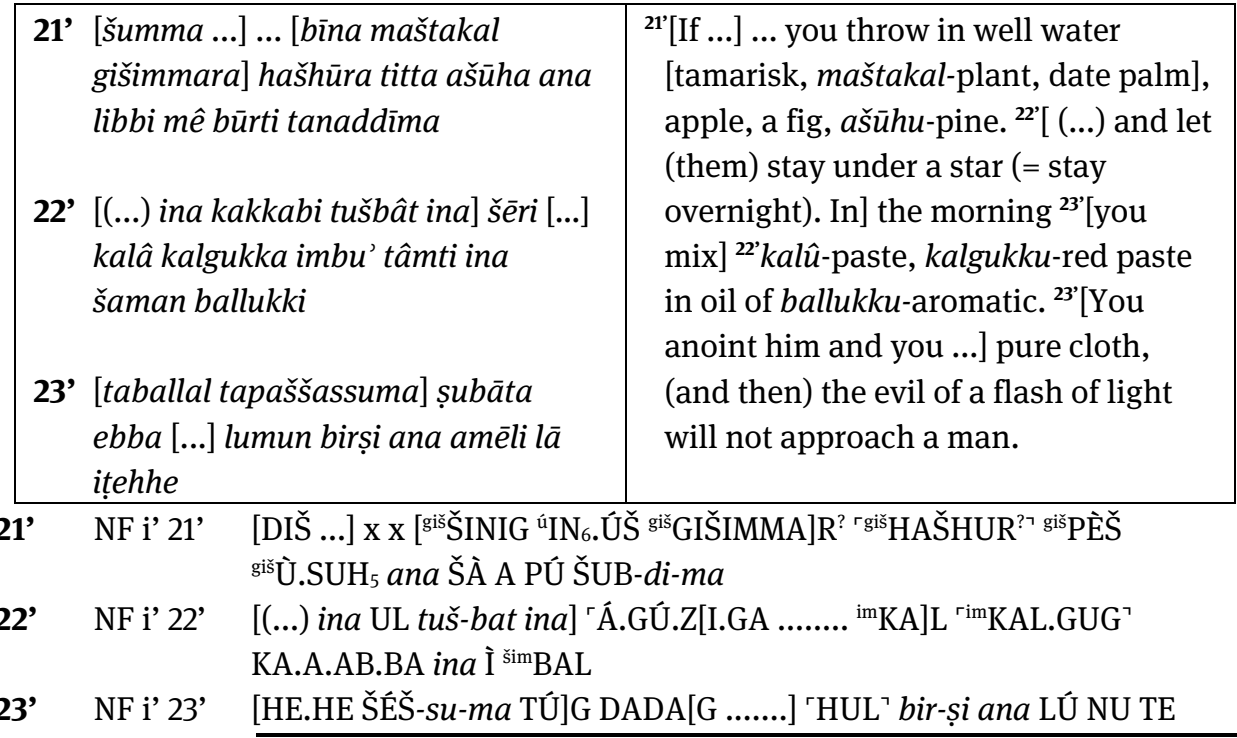

24' NF i' 24' traces

\section{BREAK OF UNKNOWN LENGTH!}

25’ NF ii' 1'

$$
\mathrm{x} \mathrm{X}[\ldots \ldots
$$

26' šumma amēlu mukīl rēš [lemutti iṣbassu ...]

27’ lū zikara lū sinništa ina ūri tušèlīma [...]
${ }^{26}$ If a supporter-of-[evil seized] a man $[. . .]^{27}$ You shall have either a man or a woman climb on to the roof, and [...]. ${ }^{28}$ 'May (it) be left behind. In the night, as soon as the sun [sets ...]. ${ }^{29}$ You set

Overview of Manuscripts and Parallels concerning NF (BAM 520): AI=LKA 145; AJ=BAM 216; AK=AO 11447; dk=Sumer 9/29; NE=BAM 516; $\mathbf{N F}=$ BAM 520 (+); NL=AMT 49/4; NM=BAM 489+508; NN=K 6329; NO=K 8211+... (+) BM 98589...; NT=AMT 85/2; NX=BAM 482; NXa=AMT 19/1; SA=IRAQ 65; uC=SpTU22+; XA=BM 123362 


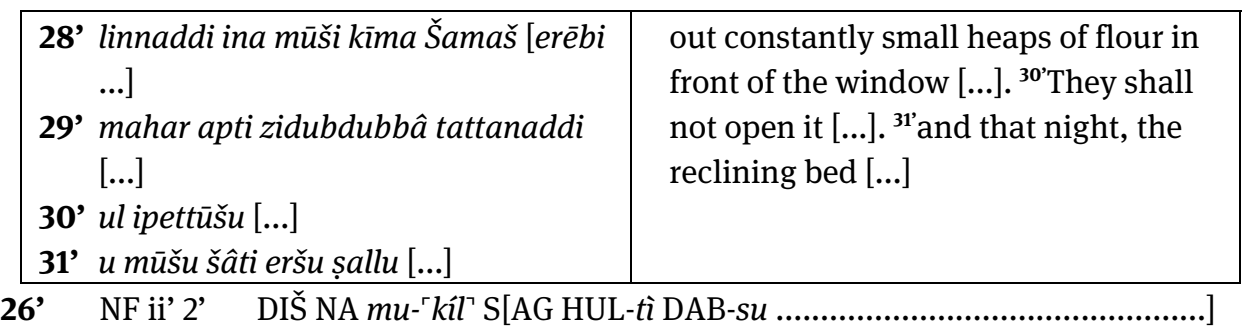

27’ NF ii’ 3’ lu NITA lu MUNUS ‘ina ÙR tu-š $e^{\urcorner}-l[i-m a$

28' NF ii' 4' li-in- $\left.{ }^{\ulcorner} n a-d i\right\urcorner$ ina $\mathrm{GE}_{6} \mathrm{GIN}_{7}{ }^{\mathrm{d}} \mathrm{UTU}{ }^{\ulcorner} e^{\urcorner}-[r e-b i$

29’ NF ii’ 5’ IGI ap-ti Zİ.DUB.DUB.BU ŠUB.ŠU[B-di

30’ NF ii' 6’ NU i-pet-tu-šu [

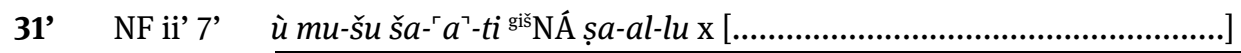

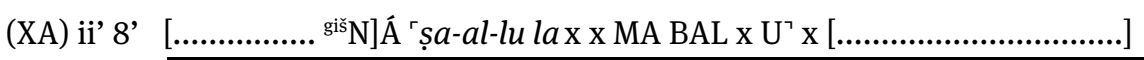

32’ šumma amēlu mukīl rēš lemutti iṣbassu mašqìta narmakta [...]

33’ ana ašri šanîmma ummu ina zumrīšu lazizma u magal ilihhib $\mathrm{x}$ [...]

34’ u zūta irašši mê bāqili u šīnāti amēlūti erēna burāša [...]

35' ina tinūri tesekker irtanahhaṣma ešeret $\mathrm{SILA}_{\mathrm{x}}(\mathrm{KISAL}) a \check{s} \bar{a} g a$... [...]
32'If a supporter-of-evil seized a man: a potion, a bath [and ...]. ${ }^{33}$ 'Alternatively: (=if a supporter-of- evil seized a man) and ummu-fever persists constantly in his body, and he growls loudly [...], ${ }^{34}$ and he sweats. ${ }^{35}$ You heat up ${ }^{34}$ maltster- water and human urine, cedar, burāšu-juniper, [tree-cone .... ${ }^{35}$ in a tannour-oven. It (the mixture) shall be continually drenched, and lyou ...] 10 SILA-measures of $a \check{a} \overline{a g u-}$ acacia [...].

32’ NF ii’ 8' DIŠ NA mu-kíl SAG HUL-tì DAB-su maš-qí-ta nar-ma-ak-t[a ................] (XA) ii' 9 $\mathrm{D}] \mathrm{AB}{ }^{\ulcorner} m a s ̌-q \hat{i}-t a$ nar? ${ }^{?}$ $[\ldots . . . . .$.

33’ NF ii’ 9' DIŠ KIMIN KÚM ina SU-šú la-zi-iz-ma u ma-gal i-li-hi-ib x [.

Overview of Manuscripts and Parallels concerning NF (BAM 520): AI=LKA 145; AJ=BAM 216; AK=AO 11447; dk=Sumer 9/29; NE=BAM 516; $\mathbf{N F}=$ BAM 520 (+); NL=AMT 49/4; NM=BAM 489+508; NN=K 6329; NO=K 8211+... (+) BM 98589...; NT=AMT 85/2; NX=BAM 482; NXa=AMT 19/1; SA=IRAQ 65; uC=SpTU22+; XA=BM 123362 
(XA) ii' 10f.' [. $\left.l] a-{ }^{\ulcorner} z i-i z-m a u^{?} m a^{?\urcorner}-g[a l] ?\ulcorner i\urcorner-[l] i-\ulcorner i b\urcorner \mathrm{KA}-\check{s} \mathfrak{u}\right\urcorner^{\urcorner}$ $\mathrm{x}[\ldots] /[\ldots] \mathrm{x} \mathrm{MA} \rightarrow$

(NL) iv 2' [... DIŠ KI]MIN KÚM 'ina SU-šú $l\left[a^{?}\right.$

34' NF ii' 10' $u$ IR TUK A SAR.MUNU 6 KÀŠ $a$-me-lu-ti giš EREN ${ }^{\text {šm } L[I ? ~}$

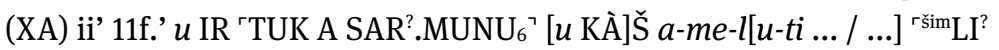
rgiššE.Ù.SUH ${ }_{5} \mathrm{NAGA}^{?} . \mathrm{SI}^{?\urcorner} \mathrm{x}[\ldots \mathrm{D}] \mathrm{U}^{?}{ }^{?}{ }^{\ulcorner} \mathrm{A}^{?\urcorner} \cdot \mathrm{BI}^{?} \rightarrow$

35' NF ii' 11' ina NININDU ÚŠ-er ir-ta-na-haṣ-ma 10? SILA $_{\mathrm{x}}(\mathrm{KISAL})^{\text {gišKIŠ }} \mathrm{K}_{16} \mathrm{x} \mathrm{x}$ [...] (XA) ii' 12f.' 'ina NININD[U ÚŠ-er / ir-ta-n] $a$ - 'haș-ma ina İ.GIŠ gišKIŠI ${ }_{16}$... ŠÉŠ-su$m a^{\urcorner}\left[\ldots \mathrm{TI}^{?}\right]$

36’ én ur-sag dasal-lú-hi igi-bi hé-p[à sag-hul-ha-za hé-pà]

37’ ur-sag ď̃à-zu igi-bi h[é-pà sag-hulha-za hé-pà] / $/{ }^{(\mathrm{AI})}$ ur-sag den-ki-ke ${ }_{4}$ kimin

38' ur-sag den-ki igi-b[i hé-pà sag-hulha-za hé-pà] / /(AI) ur-sag ďšà-zu kimin

39’ ur-sag dnin-urta i[gi-bi hé-pà saghul-ha-za hé-pà]

40' ad-da-mu / (var.) dda-mu / dumu-sag dag-ke ${ }_{4}$ gù mu-un-na-an-dé-e]

41' dumu-mu nam-tar [...] / (NO) dumumu nam ba-ši-in-tal lú igi nu-un-barra

42' lúa-zu di-k[u u $_{5}$ inim-bi nu-mu-un-tarra tú -én] / (AI) lúa-zu tag-ga [inim]-bi nu-tar-ra tu -én

43’ [ka-inim-ma] sag-hul-ha-za-[kám]
${ }^{36}$ Incantation: O hero Asalluhi, may this eye be adjured, [may the supporter-ofevil be adjured!] ${ }^{37} \mathrm{O}$ Šazu (Marduk), may this eye be adjured, [may the supporter-of-evil be adjured!] / ${ }^{(\mathrm{AI})} \mathrm{O}$ hero of Enki, ditto! ${ }^{38} \mathrm{O}$ hero (of) Enki, may this eye be adjured, [may the supporter-of-evil be adjured!] / ${ }^{(\mathrm{Al})} \mathrm{O}$ hero Šazu, ditto! ${ }^{39^{\prime}} \mathrm{O}$ hero Ninurta, may this eye be adjured, [may the supporter-of-evil be adjured!] ${ }^{40} \mathrm{My}$ father (var. Dāmu), the first-born son [of Nabû speaks to him.]/ 41' 'My son, the decision has been made (for this case): the man can not see!' ${ }^{42}$ The $a s \hat{\text { - }}$ physician (is) a judge (who) cannot [decide this case!] / ${ }^{(\mathrm{AI})}$ The attending (lit. touching) asû-physician cannot decide this [case]. ${ }^{43}$ Incantation spell. [It is an invocation against] the supporter-of-evil!

36' NF ii' 12' én ur-sag dasal-lú-hi igi-bi hé-p[à .....................................]

(NM) i’ 37' én ur-sag 'd ' [asal-

(NN) ii' 9f.' 'én ur?-sag dasal-lú-hi igi-bi hé-'pà? / sag-hul-ha-za 'hé-pà ${ }^{\prime}$

(NO) ii 10f.' én ur-sag dasal-lú-hi igi-bi hé-pà / sag-hul-ha-za hé-pà

Overview of Manuscripts and Parallels concerning NF (BAM 520): AI=LKA 145; AJ=BAM 216; AK=AO 11447; dk=Sumer 9/29; NE=BAM 516; $\mathbf{N F}=$ BAM 520 (+); NL=AMT 49/4; NM=BAM 489+508; NN=K 6329; NO=K 8211+... (+) BM 98589...; NT=AMT 85/2; NX=BAM 482; NXa=AMT 19/1; SA=IRAQ 65; uC=SpTU22+; XA=BM 123362 
(NX) iv 32' 'én ur'-sag dasal-lú-hi š[á ...]

(NXa) iv 13' [é]n ur-sag das[al-lú-hi ...]

(XA) ii' 14' [é]n ur-sag dasal-lú-hi igi-bi hé-pà sag-hul-ha-za h[é-pà]

(AI) 10 ur-sag dasal-lú-hi 'igi'-bi hé-pà sag-hul-ha-za hé-pà

(AJ) 15’ [én u]r-sag dasal-'lú'-hi ŠID-' $n u^{\urcorner}$

(AK) 19 én ur-sag dasal-lú-hi ana UGU ŠI[D ...]

(sA) i 19 én ur-sag dasal-lú-hi i-na UGU ŠID LAL

(dk) 6' hé]-pà sag-'hul'-ha-za 'hé'-pà

37' NF ii' 13' ur-sag d'̌̀̀-zu igi-bi h[é-pà

(NM) i' 38' ur-sag ďs̆à

(NN) ii' 11' ur-sag 'šà-zu igi-bi hé-pà sag-hul-h[a-

(NO) ii 12f.'ur-sag dšà-zu igi-bi hé-pà / sag-hul-ha-za hé-pà

(XA) ii' 15' [u]r-sag ď̌à-zu igi-bi hé-'pà? $\rightarrow$

(AI) 11 ur-sag den-ki-ke ${ }_{4}{ }^{~}$ kimin $^{\top}$

(dk) 7’

.] hé-pà sag-'hul'-ha-za hé-pà

38' NF ii' 14' ur-sag den-ki 'igi'-b[i hé-pà

(NM) i' 39' ur-sag den-k[i

(NN) ii' 12' ur-sag den-ki-ke 4 igi-bi hé-pà sag-hul [

(NO) ii' 14f.'ur-sag den+ki-ke 4 igi-bi hé-pà / sag-hul-ha-za hé-pà

(XA) ii' 15' ur-sag den-ki igi-bi hé-p[à]

(AI) 12 ur-sag ď̀̀-zu ki[min]

(dk) 8'

-b]i hé-pà sag-'hul'-ha-za hé-pà

39' NF ii' 15' ur-sag dnin-urta i[gi-bi hé-pà

(NM) i' 40' ur-sag dni[n-urta

(NN) ii' 13f.'ur-sag dnin-urta igi-bi h[é-pà] / sag-hul-ha-za [.........]

(NO) ii' 16f.'ur-sag 'nin-urta igi-bi hé-pà / sag-hul-ha-za hé-pà

(XA) ii' 16' ur-sag 'nin-urta 'igi-bi hé-pà $\rightarrow$

(AI) 13 ur-sag d nin-urta 'igi'-bi hé-'pà sa[g-hul-ha-za hé-pà]

(dk) 9', -b]i hé-pà sag-hul-ha-za hé-pà

(dk) 10'

(dk) 11' .]-bi hé-pà sag-hu[1-h]a-za hé-pà sa]g-hul-ha-za [hé]-pà

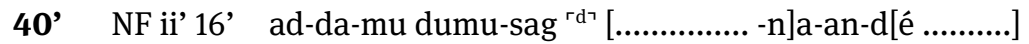
(NM) i' 41' ad-mu dumu-sag rd? [. (NN) ii' 15' da-mu dumu-sag dag-ke ${ }_{4}{ }^{\ulcorner}$gù ${ }^{\mathrm{d}} \mathrm{m}[\mathrm{u}$

Overview of Manuscripts and Parallels concerning NF (BAM 520): AI=LKA 145; AJ=BAM 216; AK=AO 11447; dk=Sumer 9/29; NE=BAM 516; $\mathbf{N F}=$ BAM 520 (+); NL=AMT 49/4; NM=BAM 489+508; NN=K 6329; NO=K 8211+... (+) BM 98589...; NT=AMT 85/2; NX=BAM 482; NXa=AMT 19/1; SA=IRAQ 65; uC=SpTU22+; XA=BM 123362 
(NO) ii' 18' dda-mu dumu-sag dag-ke 4 gù mu-un-na-an-dé-e

(XA) ii' 16' 'da-mu dumu-sag dag gù mu-un-na'-a[n-dé-e]

(AI) 14 ad-da-mu dumu-sag 'nam ${ }^{\urcorner}$-ma-kal gù $x$ [.................]

(dk) 12' g]ù mu-un-na-an-`dé??-e

41' NF ii' 17' dumu-mu nam-tar [.......................] nu-un-b[ar ....]

(NM) i' 42' dumu-mu nam-ba-š[i-

(NN) ii' 16' dumu-mu nam ba-ši-in-[......................................]

(NO) ii' 19' dumu-mu nam ba-ši-in-tal lú igi nu-un-bar-'ra' ${ }^{\top}$

(XA) ii' 17' dumu-mu nam-tar-meš [...............................] $\rightarrow$

(AI) 15 dumu-mu nam ba-ši-in-tal lú igi 'bi?` [.............]

(dk) 13' [....................................... x lú igi mi-in-bar-re

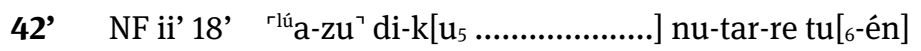

(NM) i’ 43' lúa-zu di-ku x [...........................................]

(NN) ii’ 17’ lúa-zu di-ku inim-bi n[u ...............................

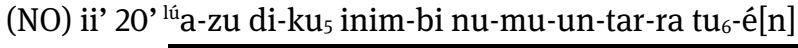

(XA) ii' $17^{\prime}[\ldots-\mathrm{z}] \mathrm{u}^{\mathrm{r}} \mathrm{di}^{\urcorner}-\mathrm{k}\left[\mathrm{u}_{5} \ldots ..\right]$

DIVIDING LINE IS PROBABLE BUT NOT TO BE SEEN!

(AI) r 1 lúa-zu tag-g[a inim]-'bi nu?-tar-ra tu[6-én]

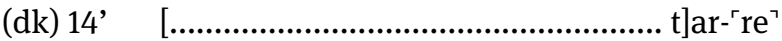

43’ NF ii’ 19' ka-inim-`ma` sag-hul-ha-za-[kám]

\begin{tabular}{|c|c|}
\hline $\begin{array}{l}\text { 44’ dudubi sebe [...] ina țurri šīpāti } \\
\text { sāmāti tašakkak [...] } \\
\text { 45’ 'imhur-lìm [...] sikillu kiškanû zēr bīni } \\
{[\ldots]} \\
\text { 46’... } \\
\text { [... talappap] ina pūtišu tarakkassu } \\
\text { [...] }\end{array}$ & 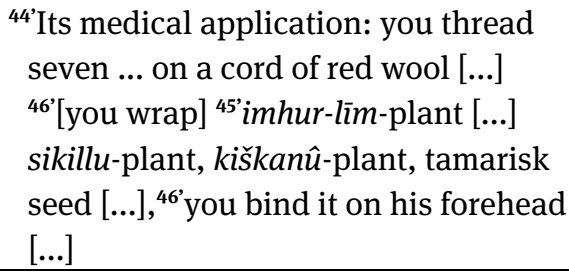 \\
\hline \multicolumn{2}{|c|}{ NF ii’ 20’ DÙ.DÙ.BI 7 x [...... i]na DUR SÍG SA È $^{\prime}-a k$ Ú x [.......] } \\
\hline \multicolumn{2}{|c|}{ 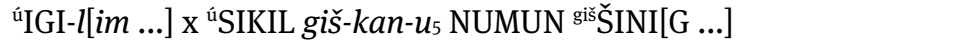 } \\
\hline \multicolumn{2}{|c|}{$\mathrm{x} \times[\ldots t a-l a-p a] p^{?}$ ina SAG.KI-šú KEŠDA- ${ }^{\ulcorner} s u^{\urcorner} \times[\ldots . .]}$. \\
\hline
\end{tabular}

Overview of Manuscripts and Parallels concerning NF (BAM 520): AI=LKA 145; AJ=BAM 216; AK=AO 11447; dk=Sumer 9/29; NE=BAM 516; $\mathbf{N F}=$ BAM 520 (+); NL=AMT 49/4; NM=BAM 489+508; NN=K 6329; NO=K 8211+... (+) BM 98589...; NT=AMT 85/2; NX=BAM 482; NXa=AMT 19/1; SA=IRAQ 65; uC=SpTU22+; XA=BM 123362 
47’ NF ii’ 23' $x$ ] $\mathrm{x} \times[$ ]

\section{§ IV.2 NK (BAM 518)}

Manuscript: $\quad$ NK (BAM 518), collated.

Museum number: $\quad$ British Museum, BM 121042.

Origin and date: Nineveh ${ }^{\text {? }} 8^{\text {th }}-7^{\text {th }}$ century BC.

Type of tablet: Fragment from the right side of possibly two-column tablet, written in a large Assyrian script. Köcher 1982b: xiii considered the text as coming from Nineveh, which is why he included it in BAM 6. However, the form of the signs and the nature of the fragment might also suggest Assur as the tablet's provenance.

Measurements: ca. $6,6 \times 7,3 \times 2 \mathrm{~cm}$.

Copy: BAM 518.

Photo/CDLI nr.: $\quad$ Plate 33/P398602.

Literature: $\quad$ Lambert and Millard 1968: 5; Köcher 1982b: xiii; Fincke 2000: 331; Scurlock and Andersen 2005: 793; Scurlock 2014: 756; Parys 2014: 20, 55ff.; Attia 2015: 38, 59.

\begin{tabular}{|l|l|l|l|}
\hline Ll. 6ff.', 9ff.' & \multirow{2}{*}{ parallels } & AB (BAM 159) iv 9f.', iv 10ff.' & § I.4 \\
\cline { 3 - 4 } L. 8' & & IGI 2, ND (BAM 515) i 10 & § II.1 \\
\hline
\end{tabular}

\section{Edition}

1' NK 1' .] $\mathrm{x} \times \mathrm{XX}$

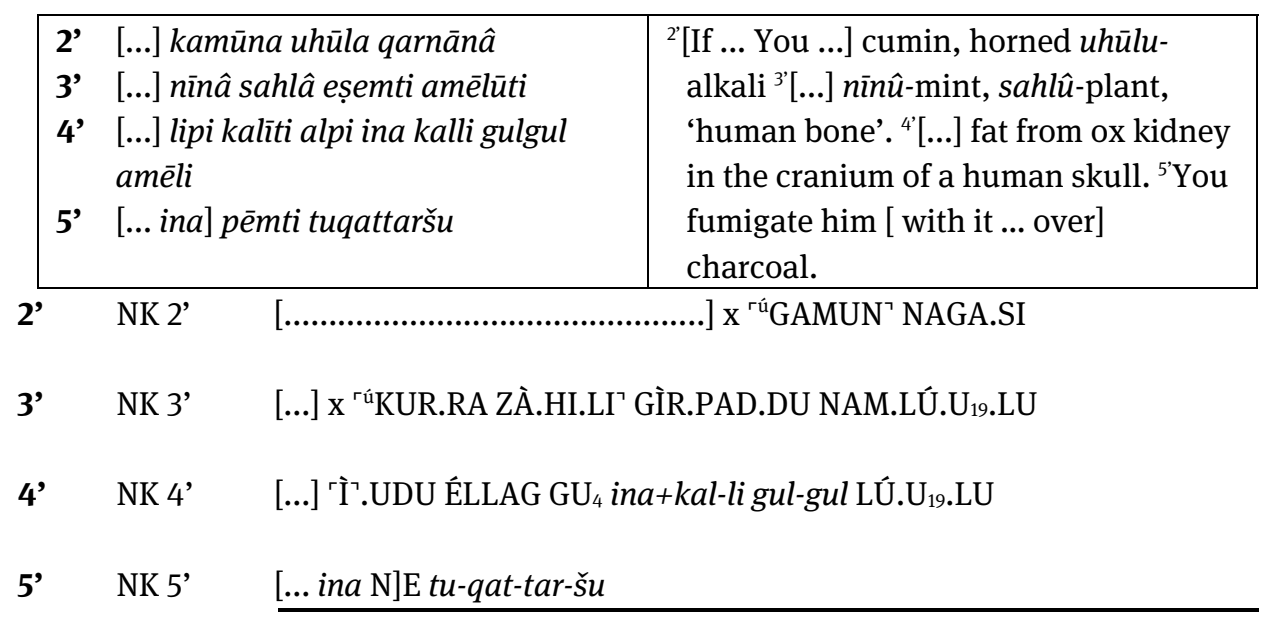




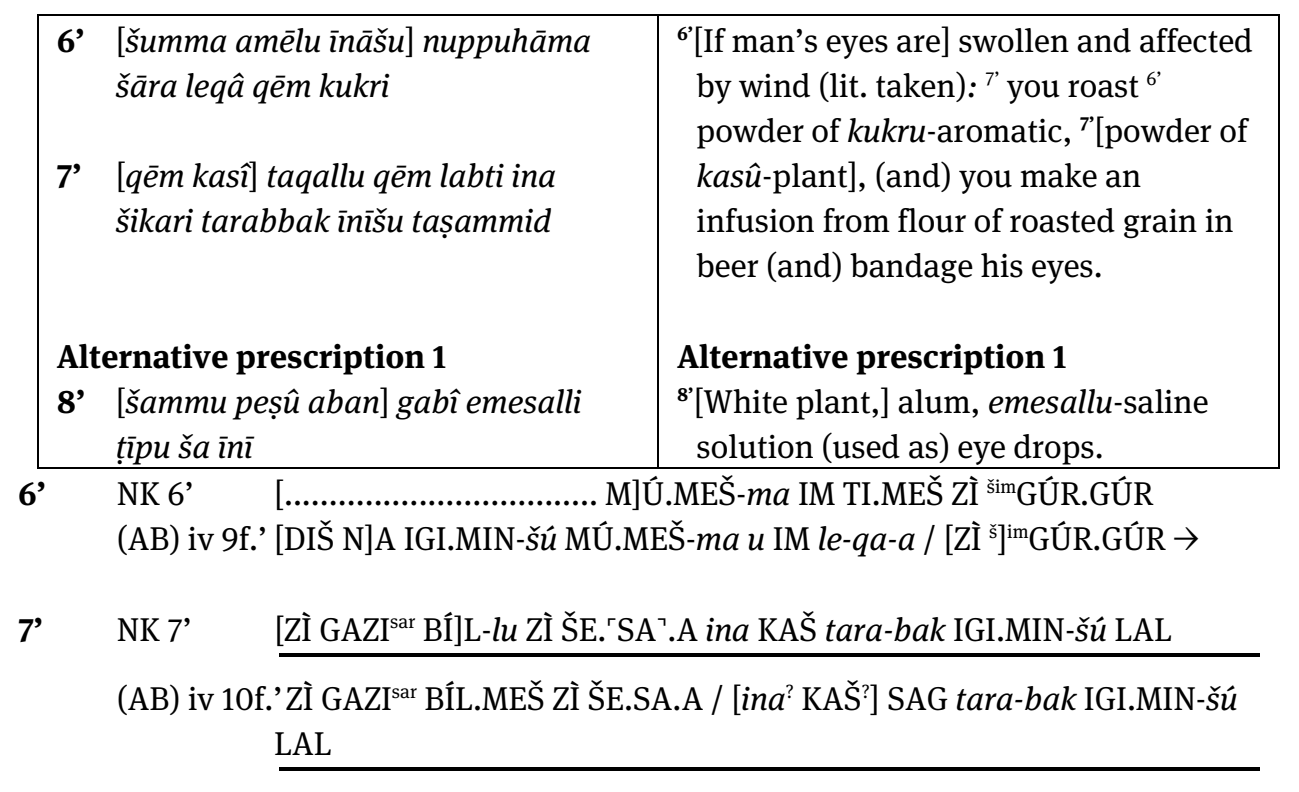

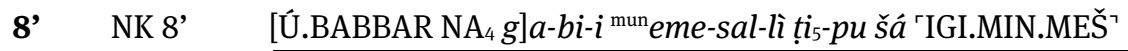

(ND) i 10 Ú.BABBAR NA 4 ga-bi-i ${ }^{\mathrm{mun}}$ eme-sal-lì an-nu-ú ti-[pu šá IGI.MIN]

More parallels in IGI 2: 10 above.

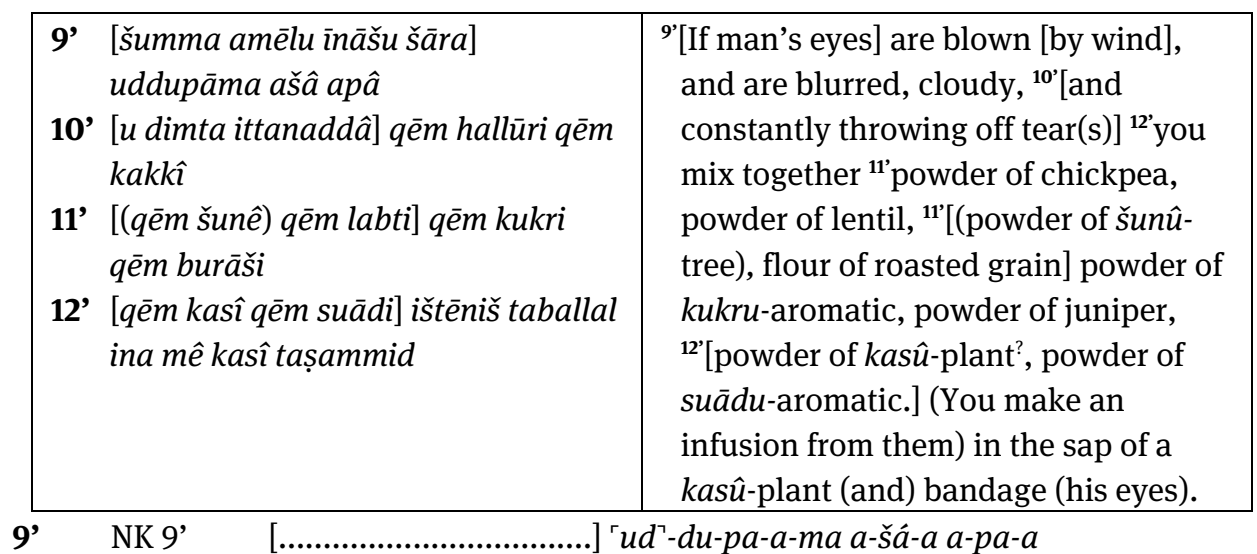

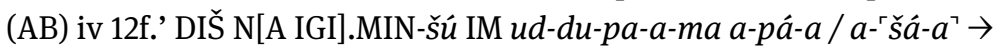

10' NK 10' ..]-` $a\urcorner$ Zİ GÚ.GAL Zİ GÚ.TUR

(AB) iv 13’ ' $u$ ' ÉR ŠUB.ŠUB- $a$ Zİ GÚ.GAL 'Zİ GÚ.TUR` 
11' NK 11' [(Zİ gišŠE.NÚ.A) Zİ ŠE.SA]. 'A A ZI šmGÚR.GÚR Zİ šmLI

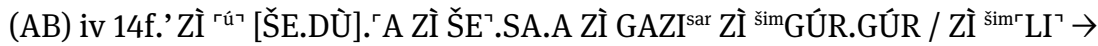

12’ NK 12' [Zİ GAZI ${ }^{\text {sar }}$ Zİ šmMAN.DU 1-n]iš HE.HE ina A GAZI ${ }^{\text {sar }}$ LAL

(AB) iv 15f.' 'ZI šim? MAN.DU 1-niš HE.HE / ina A GAZI rsar tara-bak SAG.KI.MIN-šú IGI.MIN-šú LAL-ma TI $\urcorner$

\begin{tabular}{|c|c|c|}
\hline $\begin{array}{l}13^{\prime} \\
14^{\prime} \\
15^{\prime} \\
16^{\prime} \\
17^{\prime}\end{array}$ & $\begin{array}{l}\text { [...] erbēt šiqil qēm hallūri erbēt šiqil } \\
\text { qēm kakkî } \\
\text { [...erbēt] šiqil sahlê šumma kūṣu } \\
{[\ldots . .] \text { uznīšu u ìnišu tașammid }} \\
{[\ldots . .] \text { ballukku? kukru kasû }} \\
{[\ldots]}\end{array}$ & $\begin{array}{l}{ }^{13}[. . .] \text { four shekels powder of chickpea, } \\
\text { four shekels powder of lentil, }{ }^{14}[\ldots \\
\text { four] shekels of sahlu-plant. When it is } \\
\text { winter }{ }^{15^{\prime}}[\ldots] \text { you bandage his ears and } \\
\text { eyes. }{ }^{16}[. . .] \text { ballukku-aromatic, } k u k r u- \\
\text { aromatic, kasû-plant. }{ }^{1{ }^{\prime}} \text {... }\end{array}$ \\
\hline & \multicolumn{2}{|c|}{ [........................................... $]^{r} 4^{7}$ GÍN Zİ GÚ.GAL 4 GÍN Zİ GÚ.'TUR } \\
\hline & \multicolumn{2}{|c|}{ 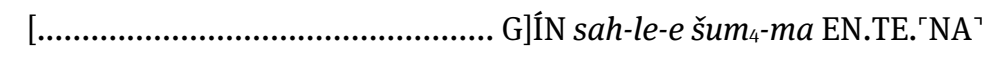 } \\
\hline & \multicolumn{2}{|c|}{ [........................................... 'GEŠ.TÚG.MIN-šú u IGI.MIN?-šú LAL } \\
\hline & \multicolumn{2}{|c|}{ [ } \\
\hline & \multicolumn{2}{|c|}{[} \\
\hline
\end{tabular}

\section{§ IV.3 NP (BAM 517)}

Manuscript: $\quad$ NP(BAM 517), collated.

Museum number: British Museum, K 10280.

Origin and date: Nineveh? ? 7 th century BC.

Type of tablet: $\quad$ Fragment from the right side of a two-column tablet. Written in big Assyrian script. As is the case with Ms. NK (BAM 518), the origin might be Assur and not Nineveh.

Measurements: $\quad$ ca. $4,7 \times 3,4 \times 2,5 \mathrm{~cm}$.

Copy: $\quad$ BAM 517.

Photo/CDLI nr.: $\quad$ Plate 33/P398602.

Literature: Köcher 1980b: xiii. 


\section{Edition}

\section{Obverse?}

\begin{tabular}{|c|c|c|}
\hline $\begin{array}{l}1^{\prime} \\
2^{\prime}\end{array}$ & $\begin{array}{l}{[\ldots] \mathrm{x}} \\
{[\ldots] \text { tasâk teqqi }}\end{array}$ & $\begin{array}{l}1^{\prime}[\ldots] \mathrm{x}^{2}[\ldots] \text { you pound (it and) daub (his } \\
\text { eyes). }\end{array}$ \\
\hline 1 ' & NP $1{ }^{\prime} \quad[\ldots .$. & 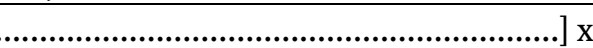 \\
\hline 2' & NP 2' & SÚ]D 'MAR \\
\hline
\end{tabular}

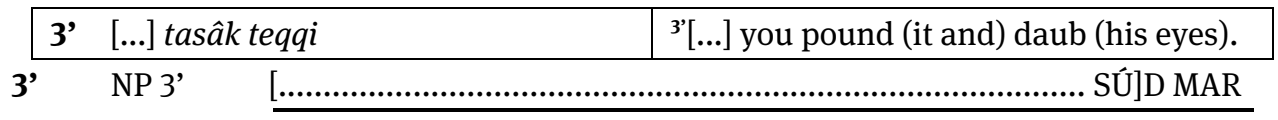

\begin{tabular}{|l|l|l|}
\hline 4' $[\ldots]$ tasâk teqqi & ${ }^{4}[\ldots]$ you pound (it and) daub (his eyes). \\
\hline
\end{tabular}

\begin{tabular}{|l|l|l|}
\hline $\mathbf{5}^{\prime}$ & {$[\ldots]$ tasâk teqqi } & ${ }^{5}[\ldots]$ you pound (it and) daub (his eyes). \\
\hline 5
\end{tabular}

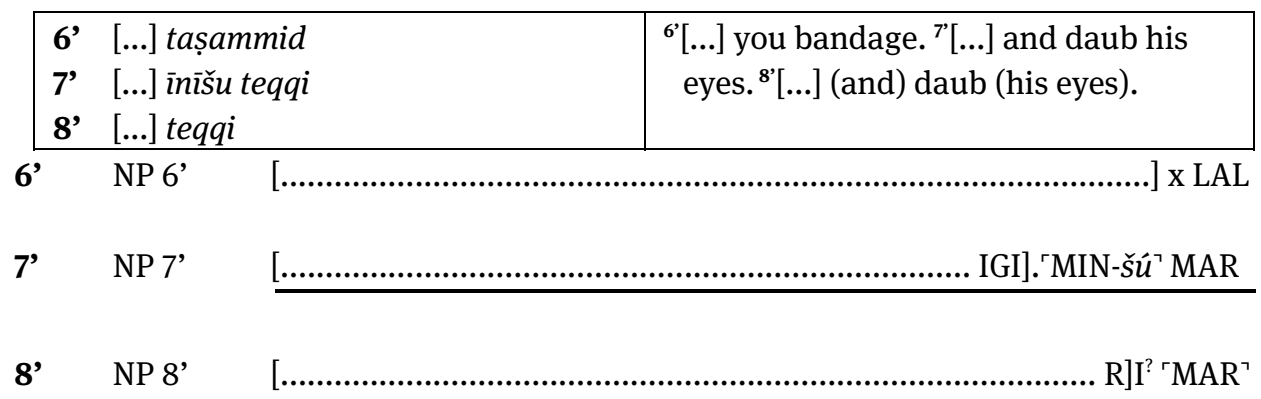

\section{Reverse?}

\begin{tabular}{|c|c|c|}
\hline $\begin{array}{l}1 \\
2^{\prime} \\
3\end{array}$ & $\begin{array}{l}{[\text { šiptu ...] izzazzu/ū }} \\
{[\ldots] \ldots} \\
{[\ldots t \hat{e}] \text { šipti }}\end{array}$ & $\begin{array}{l}{ }^{1}[\text { Incantation ... which/they] stand/s, } \\
{ }^{2}[\ldots] \ldots{ }^{3}[\ldots \text { (Incantation) }] \text { spell. }\end{array}$ \\
\hline , & NP r.?'1' [ÉN ....... & .............................. GU]B-zu \\
\hline 2' & NP r.?2' & . SI \\
\hline
\end{tabular}


3' NP r.?3' $\left.\left(\mathrm{TU}_{6}.\right)\right]{ }^{\mathrm{E}} \mathrm{E} \mathrm{N}^{\mathrm{T}}$

4' NP r. '4' $\mathrm{x}$

\section{§ IV.4 NQ (BAM 521)}

Manuscript: $\quad$ NQ (BAM 521), collated.

Museum number: British Museum, K 5954.

Origin and date: Nineveh, 7th century BC.

Type of tablet: $\quad$ Flake from the middle of a neatly written tablet in large Late Assyrian script.

Measurements: ca. $4,1 \times 4 \times 0,9 \mathrm{~cm}$.

Copy: BAM 521.

Photo/CDLI nr.: $\quad$ Plate 34/P396287.

Literature:

Köcher 1980b: xiv; Fincke 2000: 331; Scurlock and Andersen 2005: 794; Parys 2014: 20; Scurlock 2014: 756.

\begin{tabular}{|c|c|c|c|}
\hline Ll. 5', 10ff.' & \multirow{4}{*}{ parallels } & AB (BAM 159) iv 13', 17ff.' & $\S \mathrm{I} .4$ \\
\hline L. 8' & & IGI 1, NA (BAM 510) i 30’ & $\S \mathrm{I} .1$ \\
\hline Ll. 10ff.' & & NS (AMT 18/4) 1ff.' & $\S \mathrm{IV} .6$ \\
\hline Ll. 11ff. & & bB (BAM 382) 9ff. & $\S \mathrm{II} .4$ \\
\hline
\end{tabular}

\section{Edition}

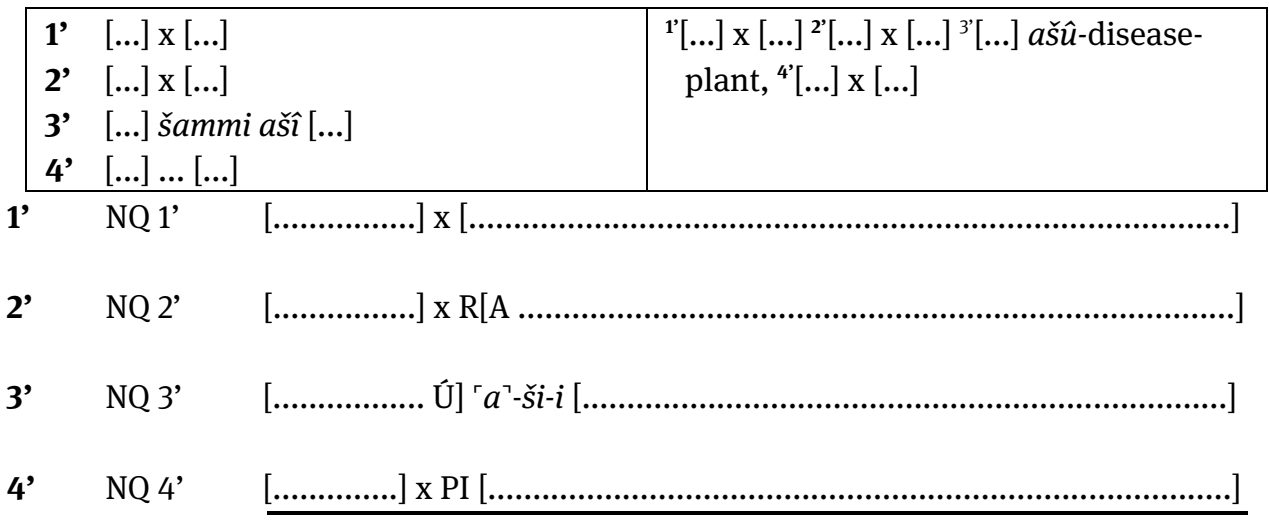




\begin{tabular}{|c|c|c|}
\hline $\begin{array}{l}5 \\
6 \\
7 \\
7\end{array}$ & $\begin{array}{l}{[\text { šumma amēlu ìnāšu abâa }] \text { ašâa } u} \\
\text { dimta ittanaddâ }[. . .] \\
{[\ldots] \text {... [...] }} \\
{[\ldots] \text { ìna marșa }[. . .]}\end{array}$ & $\begin{array}{l}5^{\prime}[\text { If a man's eyes are cloudy], blurred } \\
\text { and are constantly giving off tear(s) } \\
\left.[\ldots] .{ }^{6}[\ldots] \ldots[\ldots]\right]^{7}[\ldots .] \text { sick eyes }[\ldots]\end{array}$ \\
\hline 5' & \multicolumn{2}{|c|}{ 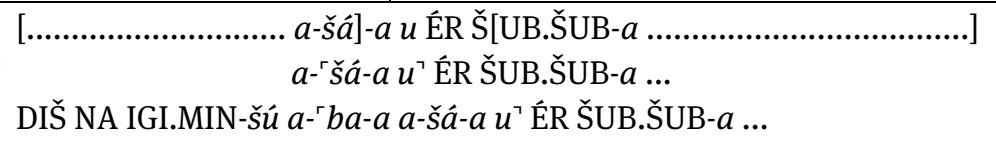 } \\
\hline 6' & {$[\ldots \ldots \ldots . .$.$] \times RI [\ldots \ldots \ldots . .$.} & 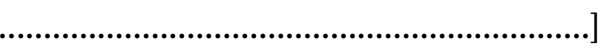 \\
\hline $7^{\prime}$ & {$[\ldots \ldots . . . \mathrm{IG}] \mathrm{I}^{?} . \mathrm{MIN}$ GIG $[$.} & 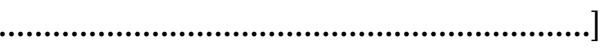 \\
\hline
\end{tabular}

\begin{tabular}{|c|c|c|}
\hline 9' & $\begin{array}{l}{[\text { šumma amēlu] īnāšu marșā sahlê }} \\
{[\text { ina šizbi tušabšal ...] }} \\
{[\ldots] \text { šuršummi šikari rūšti [...] }}\end{array}$ & $\begin{array}{l}8^{\prime}[\text { If man's] eyes are sick: [you boil] } \\
\text { sahlû-plant [in milk ...] }{ }^{9}[\ldots . .] \text { dregs of } \\
\text { premium beer [...]. }\end{array}$ \\
\hline & $\begin{array}{ll}\text { NQ 8' } & \text { [DIŠ NA IGI.MIN]-šú GIC } \\
(\text { NA) i 30’ } & {[\ldots \text { : DIŠ NA] 'IGI.MIN-šl }}\end{array}$ & 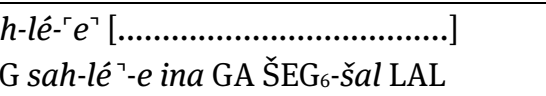 \\
\hline
\end{tabular}
more parallels in IGI 1: 35' above

9' NQ 9'

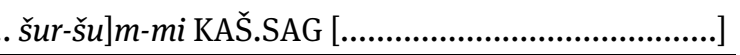

\begin{tabular}{|c|c|}
\hline 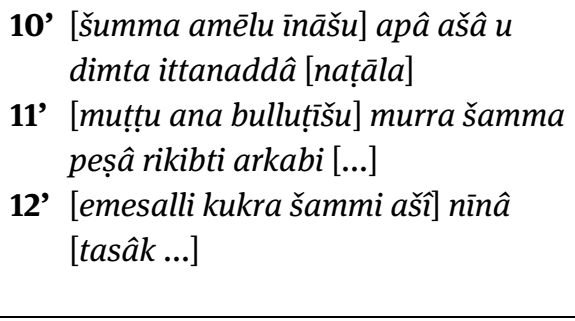 & $\begin{array}{l}{ }^{10} \text { 'If man's eyes are] cloudy, blurred, and } \\
\text { constantly giving off tear(s), [(and his) } \\
\text { seeing ability }{ }^{11} \text { 'is diminished. In order } \\
\text { to heal it: }{ }^{12 '} \text { you pound] }{ }^{11^{\prime}} \text { bitter-plant, } \\
\text { white plant, bat guano [...] }{ }^{12} \text { ' } \text { emesallu- } \\
\text { saline solution, kukru-aromatic, ašû- } \\
\text { disease-plant], nīnû-mint' [.... }\end{array}$ \\
\hline 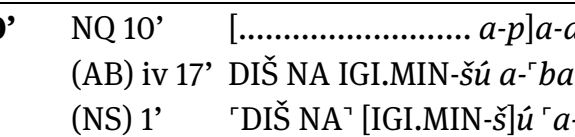 & 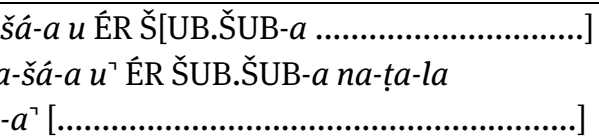 \\
\hline
\end{tabular}

11' NQ 11'

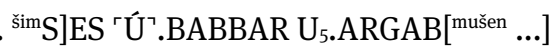
(AB) iv 18' mu-uț-ṭu ana TI-šú ršim? $S E S$ Ú.BABBAR $U_{5} . \mathrm{ARGAB}^{\text {mušen }}$
(NS) 2'
ršim? $\mathrm{SES}$ Ú.BABBAR ${ }^{\ulcorner} \mathrm{U}_{5}{ }^{?\urcorner}$. $\left[\mathrm{ARGAB}^{\text {mušen }}\right.$...]
(bB) r.1
${ }^{\text {šm }} \mathrm{SES}$ Ú.BABBAR $\mathrm{CU}_{5}{ }^{\urcorner}$. ARGAB ${ }^{\text {mušen }} \rightarrow$

12' NQ 12' ..] 'úKUR.RA? [SÚD

(AB) iv 19' ${ }^{\text {mun }}$ eme-sal-li šmGÚR. 'GÚR' Ú $a$-ši-i úKUR.RA

(NS) 3'

Ú $a$-ši-i $i$ úr KUR.RA $\urcorner$ [.

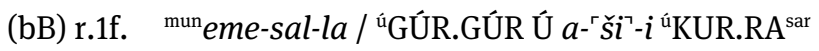




\section{§ IV.5 NR (BAM 522) and NRa (K 19831)}

Manuscript: $\quad$ NR (BAM 522), collated.

Museum number: $\quad$ British Museum, K $11695+$ K 11805.

Origin and date: Nineveh, 7th century BC.

Type of tablet: A flake from the middle of a neatly written tablet in medium large Late Assyrian script. The script is very similar to ms. NA, BAM 510 (IGI 1). On the other hand, according to Köcher 1980b: xiv fn. 22, NR might belong to ms. NE, BAM 516 (IGI 3), thus NRa (below) might be a duplicate of IGI 3 .

Measurements: ca. $5,7 \times 3,5 \times 0,5 \mathrm{~cm}$.

Copy: BAM 522.

Photo/CDLI nr.: $\quad$ Plate 34/P396287.

Literature: $\quad$ Köcher 1980b: xiv; Fincke 2000: 331; Scurlock and Andersen 2005: 794; Attia 2015: 42.

Manuscript: $\quad$ NRa (K 19831), photo-collated.

Museum number: British Museum, K 19831.

Origin and date: Nineveh, 7th century BC.

Type of tablet: $\quad$ A flake duplicating NR (BAM 522).

Photo/CDLI nr.: P404600

Literature: $\quad$ Discovered via eBL, recorded as a duplicate to NR (BAM 522) by Henry Stadhouders.

\section{Edition}

\begin{tabular}{|c|c|c|}
\hline $\begin{array}{l}1^{\prime} \\
2^{\prime}\end{array}$ & $\begin{array}{l}{[\ldots] \times x \times[. . .]} \\
{[\ldots] \text { tasâk? ìnišu teqqi ashar aban }} \\
\text { gabî }[. . .]\end{array}$ & $\begin{array}{l}{ }^{1}[\ldots] \times \mathrm{x}[\ldots]^{2}[\ldots] \text { you pound and daub } \\
\text { his eyes: ashar-stone, alum }[\ldots]\end{array}$ \\
\hline '’ & \multicolumn{2}{|c|}{ 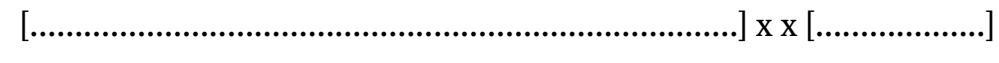 } \\
\hline ?' & \multicolumn{2}{|c|}{ 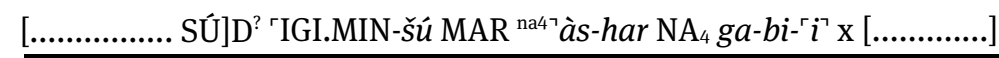 } \\
\hline
\end{tabular}

3' [šumma amēlu ìnišu dāma] ițrīma 3'[If man's eyes] oozed [blood] and it izziz marat immeri lipâ sāma ina țābti tušnâl/tuštāl

4' [...] aktam ina himēti tasâk teqqi ashar ina himēti [tasâk teqqi] stays: you cure the gall-bladder of a sheep and red fat in salt [...]. ${ }^{4}[. .$.$] You$ pound ... (and) aktam-plant in ghee (and) daub (his eyes); [you pound] ashar-stone in ghee (and) [daub (his eyes)]. 


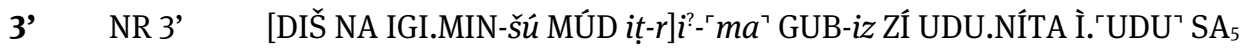
ina MUN NÁ-al x [...]

NRa 1' x x ז̀.UDU SA 5 ina MUN NÂ-al' x [...]

4' NR 4'

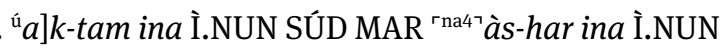
[SÚD MAR]

NRa 2'

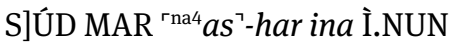
SÚD M[AR]

5’ [šumma amēlu īnišu] dāma ițrīma izziz murra ina šizbi musukkati ša zikara [ūlidu tasâk ìnī̌s teqqi]
5'[If man's eyes] oozed blood and it stayed: [you pound] bitter-plant in the milk from a woman in maternity, who [gave birth] to a male [and daub his eyes ]

5' NR 5' [DIŠ NA IGI.MIN-šú MÚ]D iṭ-ri-ma GUB-iz šm SES `ina GA ? munusÚ.ZÚG ‘̌sá NITA Ù?? [.TU SÚD IGI.MIN-šú MAR]

NRa 3' ina G]A munusú.ZÚG ša NITA ul-du ta-s[àk ...........]
6’ [šumma amēlu] īnīšu dāma țiri dalhā nīnâ šamma peșâ uhūla qarnānâ atā'iša sahlâ [...]
7’ [... aban] gabî kammu ištēniš tamarraq šumma kūṣu ina šikari tasappan ...
8' [... ina] mê temessi talâš ina tinūri teppi tušellâmma ina himēti? [...]
9' [...] tābīla [...]
${ }^{6}$ [If] blood oozed [(from) man's] eyes (and) they are troubled: ${ }^{7}$ you grind together ${ }^{6}$ 'nīnu-mint, white plant, horned uhūlu-alkali, atā'išu-plant, sahlû-plant ... ${ }^{7}[. .$.$] alum (and) kammu-$ tanning-fungus. When it is winter you [soften (it)] in beer, ${ }^{8}[$ (and) when it is summer in ...]. You wash [(it) in] water, knead (it, and) bake (it) in a tannour-oven. You remove (it), and you $[. .$.$] in ghee? [. . .]^{9}[\ldots]$ in a dry state $[\ldots]$
6' NR 6' [DIŠ NA IGI.M]IN-šú MÚD ți-ri LÙ.MEŠ 'KUR.RA? 'Ú? NAGA.SI 'úKUR ${ }^{\text {K }}$ KU[R ...]
NRa 4' ] 'Ú?'BABBAR

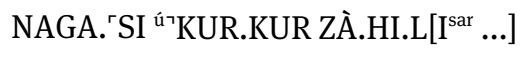

7' $\quad \mathrm{NR} 7{ }^{\prime} \quad[. . . \mathrm{N}] \mathrm{A}_{4}$ ga-bi-i kam-mu 1-niš ta-mar-ra $[q$ š $]$ um 4 -ma EN.TE.NA ina 'KAŠ` [...] 
NRa 5', EN.T]E. ${ }^{N A}$ ' ina KAŠ t[a-sa-pan ...]

8' NR 8' [... ina $]^{\ulcorner} \mathrm{A}^{\urcorner}$LUH SILA ${ }_{11}-a$ š ina NININDU te- ${ }^{\ulcorner}$pi $\mathrm{E}_{11}{ }^{\urcorner}-$ma ina ${ }^{\ulcorner} \mathrm{I}^{\urcorner} . \mathrm{N}\left[\mathrm{UN}{ }^{?}\right.$...] $]$ NRa 6, [...] x x [...]

9' NR 9' ta-bi-l] $a_{12} ?$

\begin{tabular}{|c|c|c|}
\hline & $\begin{array}{l}\text { [šumma amēlu īnišsu dāma] ițrīma } \\
\text { izziz [...] }\end{array}$ & $\begin{array}{l}{ }^{10} \text { [If man's eyes] oozed [blood] and it } \\
\text { stays [...] }\end{array}$ \\
\hline & \multicolumn{2}{|c|}{ [DIŠ NA IGI.MIN-šú MÚD i]ț-ri-ma G[UB-iz .........................................] } \\
\hline
\end{tabular}

\begin{tabular}{|c|c|}
\hline 11’ $[. .$.$] kasû-plant [...]$ & ${ }^{11}[. .$.$] kasû-plant [...]$ \\
\hline
\end{tabular}

\section{§ IV.6 NS (AMT 18/4)}

Manuscript: $\quad$ NS (AMT 18/4), collated.

Museum number: British Museum, K 9555.

Origin and date: Nineveh, 7th century BC.

Type of tablet: $\quad$ Flake from the left side of a one or possibly two-column tablet.

Measurements: $\quad 3,2 \times 4,7 \times 1,5 \mathrm{~cm}$.

Copy:

AMT $18 / 4$.

Photo/CDLI nr.: $\quad$ Plate 34/P398184.

Literature:

Köcher 1980b: xiii; Fincke 2000: 331; Scurlock and Andersen 2005: 779; Parys 2014: 20; Scurlock 2014: 751; Attia 2015: 75, 82.

\begin{tabular}{|l|l|l|l|}
\hline \multirow{2}{*}{ Ll. 1ff.' } & NQ (BAM 521) 10ff.' & § IV.6 \\
\cline { 4 - 4 } Ll. 1ff.' & \multirow{4}{*}{ parallels } & AB (BAM 159) iv 17ff.' & § I.4 \\
\cline { 3 - 4 } & & bB (BAM 382) 9ff. & § II.4 \\
\cline { 3 - 4 } Ll. 2ff. & & IGI 3, NE (BAM 516) ii 6f.' & § III.1 \\
\hline Ll. 6ff.' & &
\end{tabular}

\section{Edition}

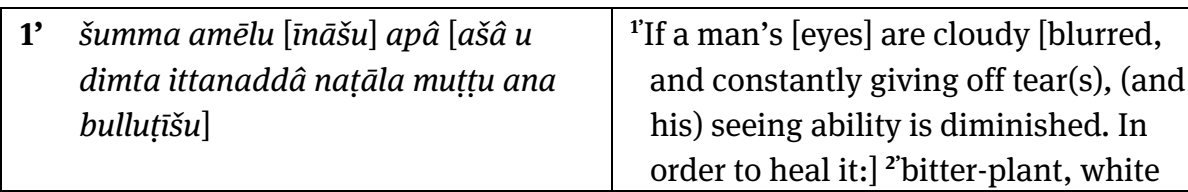




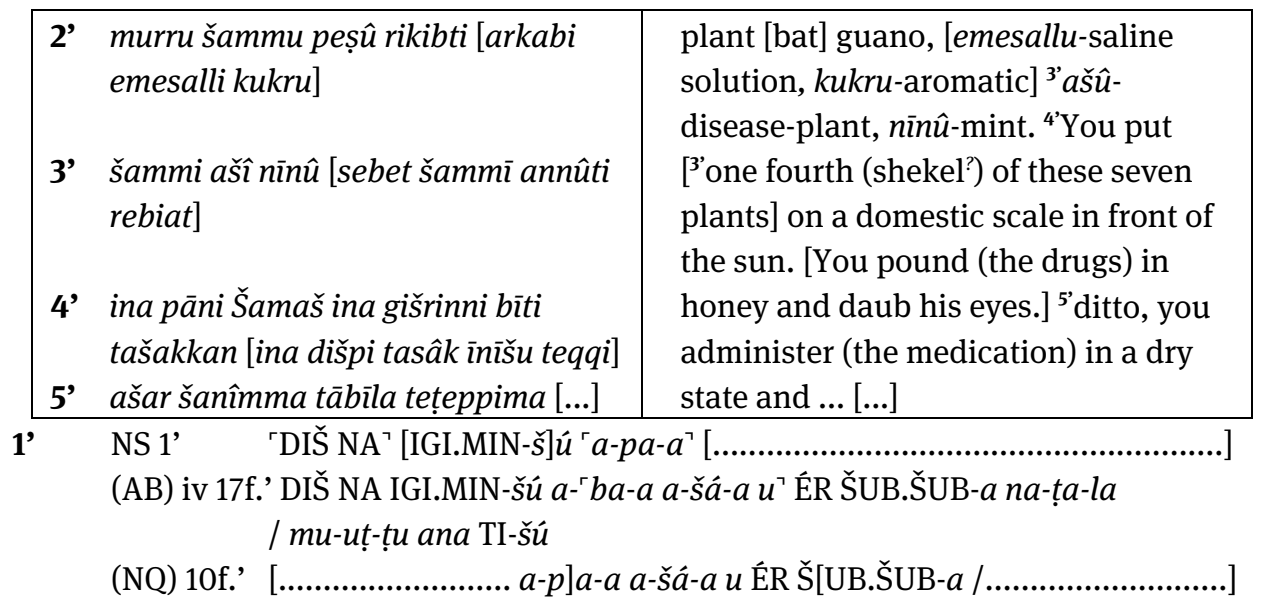

2' NS 2' ršim? $\mathrm{NES} \mathrm{Ú.BABBAR} \mathrm{U}_{5}{ }^{?\urcorner}$. [ARGAB ${ }^{\text {mušen }}$

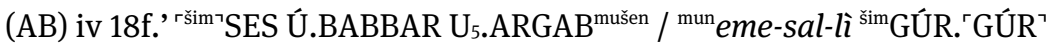

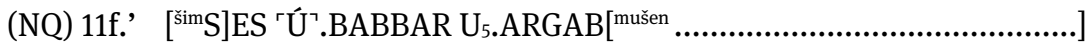

(bB) r.1f. šmsES Ú.BABBAR ' $\mathrm{U}_{5}{ }^{\urcorner}$.ARGAB ${ }^{\text {mušen mun }}$ eme-sal-la / "GÚR.GÚR

3' NS 3' Ú $a$-ši-i úr KUR.RA [

(AB) iv 19f.' Ú $a$-ši-i 'í KUR.RA / 7 Ú.HI.A ŠEŠ IGI.4.GÁL.LA.'TA'.ÀM

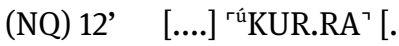

(bB) r.2 Ú $\left.a-{ }^{\ulcorner} \check{s} i\right\urcorner-i$ úKUR.RA ${ }^{\text {sar }}$

4' NS 4' ina IGI dUTU ina GIŠ.ERÍN É GAR [................................................] (AB) iv 20f.' ina IGI dUTU? / ina GIŠ.ERÍN LAL-al ina LÀL SÚD 'IGI’.MIN-šú MAR

5) NS 5, KIMIN ta-bi-la te-tep-pi- ${ }^{\circ} m a^{\urcorner} \times[$.

\begin{tabular}{|c|c|c|}
\hline 6' & $\begin{array}{l}\text { šumma amēlu īnāšu lā inațtalāa } \\
\text { amēlu [šù șēta hamiț ištēn šiqil } \\
\text { rikibti arkabi] } \\
\text { mišil šiqil šamma peșâ rebiat } \\
\text { [emesalli ina dišip šadê] } \\
\text { u himēti tasâk ... [īnišsu teqqi ...] }\end{array}$ & $\begin{array}{l}{ }^{6} \text { If a man's eyes cannot see: [that] man } \\
\text { [is inflamed with sun heat]: }{ }^{8} \text { you } \\
\text { pound [ }{ }^{\prime} \text { one shekel of bat guano], } \\
{ }^{7} \text { half shekel of white plant, (and) one } \\
\text { fourth (shekel) of [emesallu-saline } \\
\text { solution in mountain honey] }{ }^{8} \text { and in } \\
\text { ghee, ... [you daub his eyes (with it) ...] }\end{array}$ \\
\hline & $\begin{array}{ll}\text { NS 6’ }^{\prime} & \text { DIŠ NA IGI.MIN-šú la } \\
(\mathrm{NE}) \text { ii 6’ } & \text { DIŠ NA IGI.MIN-šú la } \\
& \text { U }_{5 . A R G A B}{ }^{\text {mušen }}\end{array}$ & $a-l a$ N[A BI ....................................................] \\
\hline
\end{tabular}

7’ $\quad$ NS 7’ 1/2 GÍN Ú.'BABBAR? IGI.4.GÂL.[LA ..] 
(NE) ii 7’ 1/2 GÍN Ú.BABBAR IGI.4.GÁL.LA ${ }^{\text {mun }}$ eme-sal-lì ina 'LÀL ${ }^{`}$.KUR $\rightarrow$

8’ $\quad$ NS 8' $u$ İ.NUN S[ÚD] $x$ [

NO DIVIDING LINE TO BE SEEN!

(NE) ii 7’ u İ.N[UN SÚD I]GI.MIN-šú MAR

\section{§ IV.7 NT (AMT 85/2)}

Manuscript: $\quad$ NT (AMT 85/2), collated.

Museum number: $\quad$ British Museum, K 2557.

Origin and date: Nineveh, 7th century BC.

Type of tablet: Flake from the left side of one or possibly two-column tablet.

Measurements: ca. $5 \times 3 \times 2,8 \mathrm{~cm}$.

Copy: $\quad$ AMT 85/2.

Photo/CDLI nr.: $\quad$ Plate 34/P394510.

Literature: $\quad$ Stol 1993: 73 fn. 63; Fincke 2000: 328; Scurlock and Andersen 2005:

782; Scurlock 2006: nos. 122, 129, 130 and 303; Scurlock 2008b: 197.

\begin{tabular}{|l|l|l|l|}
\hline \multirow{2}{*}{ L . 4' } & \multirow{3}{*}{ parallels } & NF (BAM 520) i' 13' & § IV.1 \\
\cline { 3 - 4 } & & uC (SpTU 22+85) i 18f. & § IV.1.3 \\
\cline { 3 - 4 } & NF (BAM 520) i' 15' & § IV.1 \\
\hline \multirow{2}{*}{ L. 6' } & &
\end{tabular}

\section{Edition}

\begin{tabular}{|c|c|c|}
\hline $\begin{array}{l}\text { 1' } \\
2^{\prime}\end{array}$ & $\begin{array}{l}\text { ittix } x[\ldots] \\
\text { u minât šêri }[\ldots] \\
\text { ina abi u țebēti teppušma ... [...] }\end{array}$ & $\begin{array}{l}\ldots{ }^{\prime} \text { 'with }[\ldots]{ }^{\prime} \text { 'and parts of [his] flesh }[\ldots] \\
{ }^{\prime} \text { You perform (it) in the month Abu (5) } \\
\text { and the month Tebet (10) and ... [...] }\end{array}$ \\
\hline & \multicolumn{2}{|c|}{ 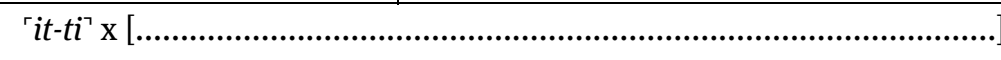 } \\
\hline & \multicolumn{2}{|c|}{ 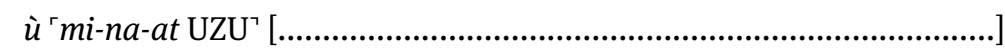 } \\
\hline & \multicolumn{2}{|c|}{ 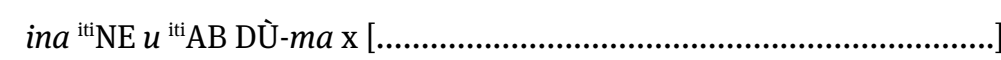 } \\
\hline
\end{tabular}

\begin{tabular}{|c|c|}
\hline $\begin{array}{l}\text { 4’ Šumma amēlu ina ìnišsu birṣī } \\
\text { [ïtanammar ...] } \\
\text { 5, ețem harbāti murtappidu [...] }\end{array}$ & $\begin{array}{l}{ }^{4} \text { If a man [constantly sees] flashes of } \\
\text { light in his eyes [and ...] }{ }^{5} \text { a roving } \\
\text { ghost of abandoned places [afflicts } \\
\text { him ...]. }{ }^{6} \text { You take }{ }^{5}[\text {... (a plant)] }\end{array}$ \\
\hline
\end{tabular}




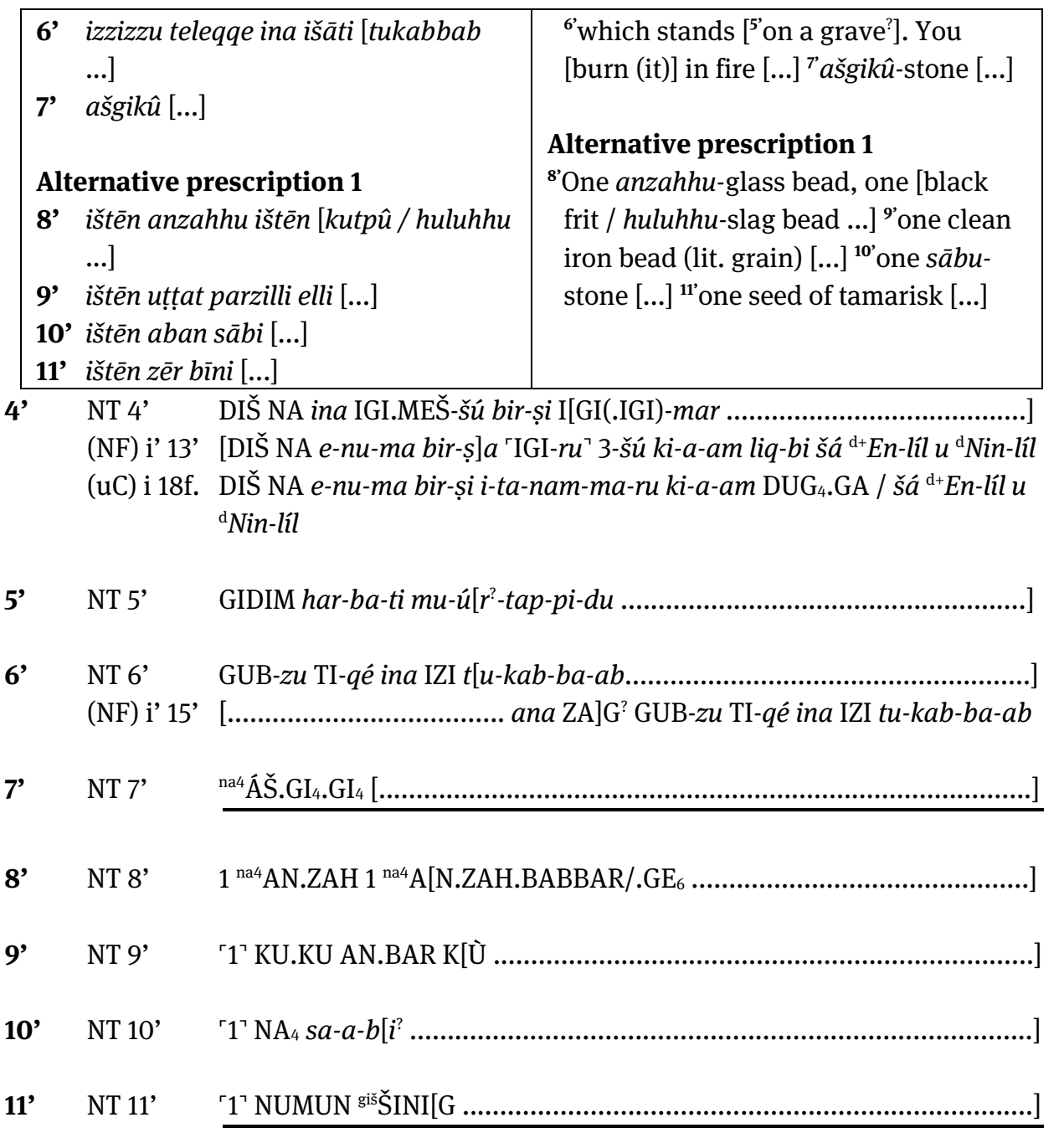

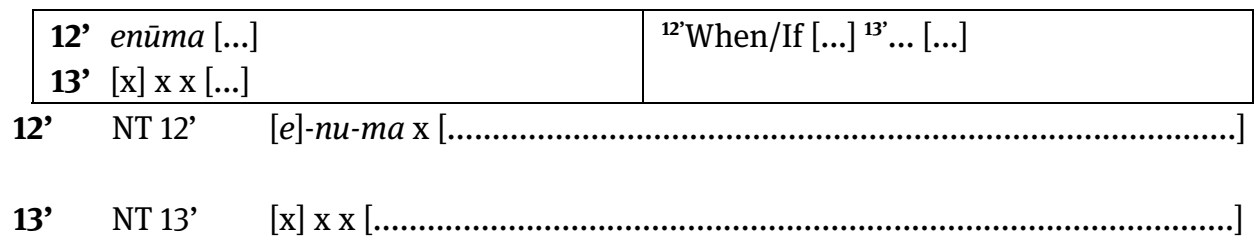




\section{$\S$ IV.8 NU (AMT 14/3)}

Manuscript: $\quad$ NU (AMT 14/3), collated.

Museum number: British Museum, K 5415a.

Origin and date: Nineveh, 7 th century BC.

Type of tablet: $\quad$ Flake from the middle part of a one or possibly two-column tablet.

Measurements: $\quad$ ca. $7,2 \times 8 \times 2 \mathrm{~cm}$.

Copy:

AMT 14/3.

Photo/CDLI nr.: $\quad$ Plate 35/P396018.

Literature:

Thompson 1926: 47f.; Farber 1974: 89; Kinnier Wilson 2011: 19.

\section{Edition}

1' NU 1'

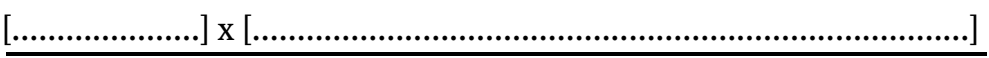

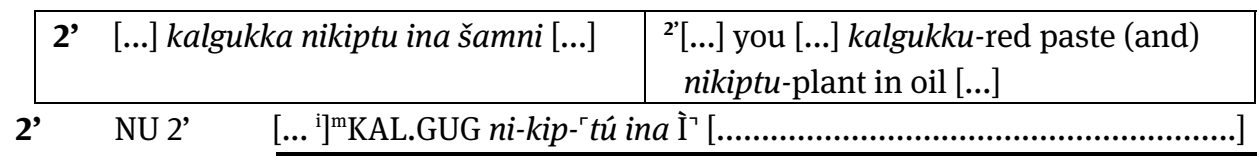

\begin{tabular}{|c|c|c|}
\hline & $\begin{array}{l}{[. . .] \text { burāša kukra imbu' tâmti }} \\
\text { šamma ēda tasâk ina tangussi [...] }\end{array}$ & $\begin{array}{l}{ }^{3}[. . .] \text { you pound }[. . .], \text { juniper, } k u k r u- \\
\text { aromatic, algae, (and) single plant. } \\
\text { [You ...] in copper tangussu-kettle [...] }\end{array}$ \\
\hline & \multicolumn{2}{|c|}{$\left[\ldots{ }^{\text {si }}\right]^{\mathrm{m}}$ LI ${ }^{\text {šm }}$ GÚR.GÚR KA.A.AB.BA Ú.DILI SÚD ina ${ }^{\text {rurudu}}{ }^{\text {Š }}[$ EN.TUR ..... $]$} \\
\hline
\end{tabular}

\begin{tabular}{|c|c|c|}
\hline & $\begin{array}{l}{[. . .] \text { hašû imhur-līm imhur-ešrā }} \\
\text { tarmuš ina kišādī̌su tašakkan [...] }\end{array}$ & $\begin{array}{l}{ }^{4}[\ldots] \text { : you place }[(\ldots)], \text { hašû-thyme, } \\
\text { imhur-lìm-plant, imhur-ešrā-plant, } \\
\text { tarmuš-lupine on his neck, [...] }\end{array}$ \\
\hline & \multicolumn{2}{|c|}{ 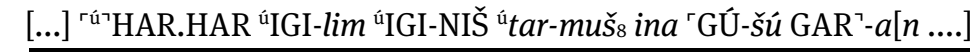 } \\
\hline
\end{tabular}

\begin{tabular}{|c|c|c|}
\hline & $\begin{array}{l}\text { [...] zalāqu tasâk ina libbi šamni } \\
\text { tanaddi libbi īnišu tapaššašma } \\
\left.\text { [iballut! }{ }^{?}\right]\end{array}$ & $\begin{array}{l}{ }^{\prime}[. . .] \text { you pound [...], (and) zalāqu-stone, } \\
\text { you put (them) into sesame oil, and } \\
\text { you smear the inner part of his eyes } \\
\text { with it, then [he should recover?.] }\end{array}$ \\
\hline & NU 5 & -GIŠ ŠUB-di ŠÀ IGI.MIN-šú ŠÉŠ-m[a? TI?] \\
\hline
\end{tabular}

\begin{tabular}{|l|l|}
\hline 6' $\begin{array}{l}\text { [...] teqqi mê bīni UD.20.KAM ìnīšu } \\
\begin{array}{l}\text { temessīma mê binni šalāšššu } \\
\text { irammukma iballuṭ }\end{array}\end{array}$ & $\begin{array}{l}{ }^{6}[. . .] \text { you daub (his eyes). For twenty } \\
\text { days, you wash his eyes with tamarisk } \\
\text { sap, then he bathes three times with }\end{array}$ \\
\hline
\end{tabular}




\begin{tabular}{|l|l|}
\hline & \multicolumn{1}{l|}{$\begin{array}{l}\text { the tamarisk sap, then he should } \\
\text { recover. }\end{array}$} \\
\hline
\end{tabular}

\begin{tabular}{|ll|l|}
\hline $7>\begin{array}{l}\text { [...] arqūssu tasâk mêšu taṣahhat ina } \\
\text { kakkabi tušbât ašar šanîmma }\end{array}$ & $\begin{array}{l}7^{\prime}[. . .] \text { you pound [...] while still green, you } \\
\text { press its sap out, let it stay under a star } \\
\text { (= stay overnight), ditto (= he should } \\
\text { recover). }\end{array}$ \\
\hline
\end{tabular}

\begin{tabular}{|c|c|c|}
\hline 8' & $\begin{array}{l}\text { [...] ana bulluți aban mūṣi mê bīni ša } \\
\text { ina pēmti tašakkanuma } \\
\text { [... ina] libbi tušeș̦i šamna lipi } \\
\text { eșemti șabìti ištēniš tasâk teqqi }\end{array}$ & $\begin{array}{l}8^{\prime}[. . .] \text { in order to heal (it): mūṣu-stone } \\
\text { (and) tamarisk sap, which you put } \\
\text { over charcoal and then }{ }^{9}[. . .] \text { you } \\
\text { remove (it) [from the] middle (of a } \\
\text { vessel). You pound together oil and } \\
\text { gazelle bone marrow (and) daub (his } \\
\text { eyes). }\end{array}$ \\
\hline 8' & \multicolumn{2}{|c|}{$[\ldots a] n a$ TI ${ }^{\text {nal}} m u-s ̦ i$ A.MEŠ gišŠINIG šá ina NE GAR-nu-m[a] } \\
\hline 9' & \multicolumn{2}{|c|}{ 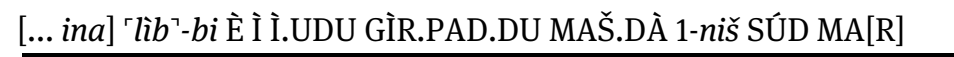 } \\
\hline
\end{tabular}

\begin{tabular}{|c|c|}
\hline $\begin{array}{l}\text { 10’ [...] kabūt Šeriš hašâ atā’ iša ina itqi } \\
\text { talammi ina kišādišu tašakkan (...)] }\end{array}$ & $\begin{array}{l}\text { 10'}^{\prime}[. . .] \text {. You wrap up ox dung, hašû- } \\
\text { thyme (and) atā'išu-plant in a woollen } \\
\text { tuft, (and) you place (it) on his neck } \\
\text { (...). }\end{array}$ \\
\hline \multicolumn{2}{|c|}{ 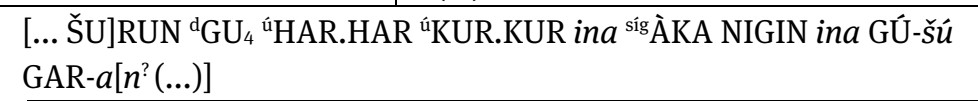 } \\
\hline
\end{tabular}

11' [...] lū mukīl rēš lemutti lū

12' [...lū ețemmu] lemnu iṣbassuma ana bulluțǐšu

13' [...] ... teleqqe ina țurri šì āti peșâti tašakkak šuruš ēri [...]

14' [...] maštakal qilip šūmī šammu ēdu kibrītu uhūlu qarnānû sebet šammī [annûti]

15' [...] ina itqi talappap èma lippi [talpap]
11'[If, ...] either the supporter-of-evil, or $[(\ldots)]^{12}[\ldots$, or] evil [ghost] afflicts him: in order to heal (him): ${ }^{13}$ [...] you take [...], (and) thread white wool on a cord. Root of êru-tree [... ${ }^{14}[$ [... maštakalplant, peel of garlic, single plant, sulphur, horned uhūlu-alkali, [these $e^{\text {? }}$ seven drugs ${ }^{15}$ [...] You wrap [...] in a woollen tuft, wherever you [wrap] a wrapping, ${ }^{16}[. .$.$] You shall recite [the$ 


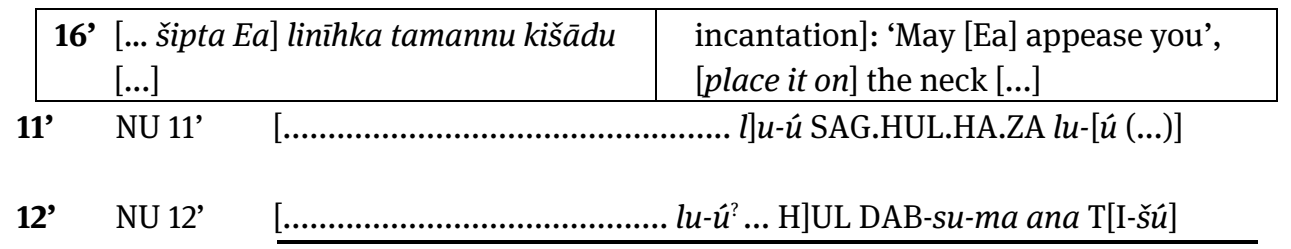

13’ NU 13' [..........] x TI-qé ina DUR SÍG BABBAR È- $a k$ SUHUŠ gišMA.[NU ...........]

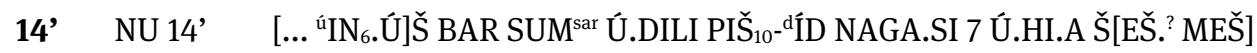

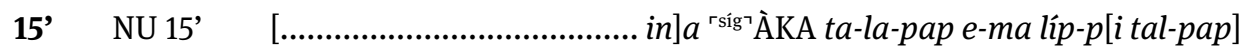

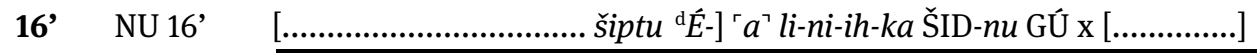

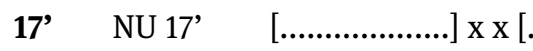

\section{§ IV.9 NV (AMT 18/3)}

Manuscript: $\quad$ NV (AMT 18/3), collated.

Museum number: British Museum, K 10535.

Origin and date: Nineveh, 7th century BC.

Type of tablet: $\quad$ Flake from the right part of a tablet/column inscribed in Babylonian Hand.

Measurements: $\quad$ ca. 4,3 $33,4 \times 0,1-1,1$ (towards the middle) $\mathrm{cm}$.

Copy: $\quad$ AMT $18 / 3$.

Photo/CDLI nr.: $\quad$ Plate 35/P238920.

Literature: $\quad$ Thompson 1926: 52; Scurlock and Andersen 2005: 779.

\begin{tabular}{|l|l|l|l|}
\hline Ll. 6f.' & parallels & NX (BAM 482) ii 62f.' & § V.2. \\
\hline
\end{tabular}

\section{Edition}

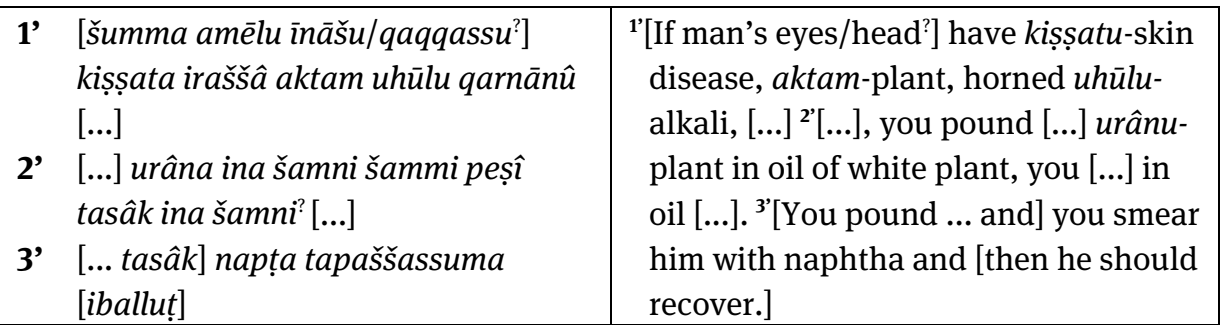


1' NV 1' [DIŠ NA IGI.MIN-šú/SAG.DU-su ki-i]ṣ-ṣa-ta ${ }_{5}$ TUK '́a $a$-tam NAGA.S[I ...]

2' NV 2'

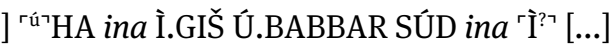

3' NV 3' SÚ]D İ.KUR.RA ŠÉŠ-su-ma [TI]

\begin{tabular}{|c|c|c|}
\hline $\begin{array}{l}4^{\prime} \\
5\end{array}$ & $\begin{array}{l}\text { [...] murra burāša tasâk ina [...] } \\
\text { [...]... UD.14.KAM taṣammid [...] }\end{array}$ & $\begin{array}{l}{ }^{4}[\ldots], \text { you pound }[\ldots], \text { bitter-plant (and) } \\
\text { juniper, }\left[\text { you ...] in }[\ldots]^{5}[\ldots] . . . \text { You }\right. \\
\text { bandage (his eyes) for fourteen days } \\
{[\ldots]}\end{array}$ \\
\hline & NV 4' & 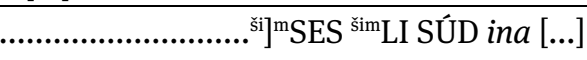 \\
\hline & {$[\ldots \ldots \ldots \ldots \ldots \ldots$} & 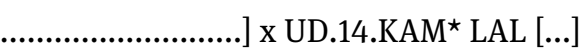 \\
\hline
\end{tabular}

\begin{tabular}{|c|c|c|}
\hline 6' & $\begin{array}{l}\text { [šumma amēlu nakkapti imittī̌̌u } \\
\text { ikkalšu]ma īn imittī̌su naphat u } \\
\text { dimta [inaqqi] } \\
\text { [šugidimmak šani] Ištar ana } \\
\text { bulluțī̌su sīhu [...] }\end{array}$ & $\begin{array}{l}\text { 6'[If man's right temple hurt him] and } \\
\text { his right eye is inflamed and [pours } \\
\text { out] tear(s). ?'[It is the 'Hand of the } \\
\text { Ghost' as a deputy of Ištar.] In order to } \\
\text { heal it: [you mix] sìhu-plant [...] }\end{array}$ \\
\hline ' & \multicolumn{2}{|c|}{ 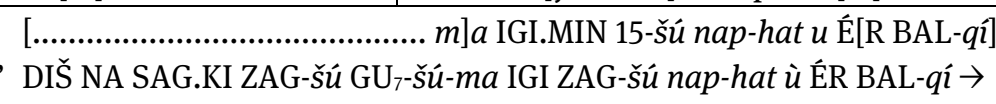 } \\
\hline $7{ }^{\prime}$ & 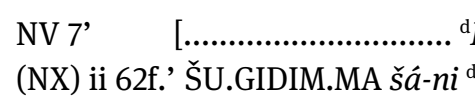 & 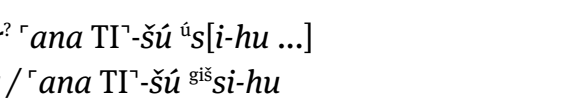 \\
\hline
\end{tabular}

\section{§ IV.10 NW (BAM 439)}

Manuscript: $\quad$ NW (BAM 439), collated.

Museum number: British Museum, K 13474.

Origin and date: Nineveh, 7 th century BC.

Type of tablet: $\quad$ Flake from the middle part of a tablet.

Measurements: $\quad$ ca. $3,1 \times 2,6 \times 1 \mathrm{~cm}$.

Copy: $\quad$ BAM 439.

Photo/CDLI nr.: $\quad$ Plate 35/P400318.

Literature: $\quad$ Köcher 1980a: no. 439.

\section{Edition}

1' NW 1'

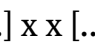




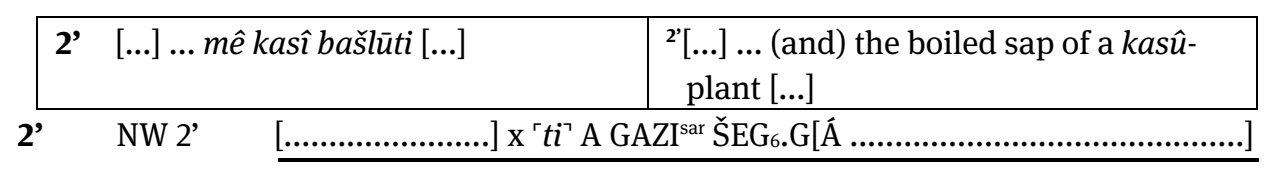

\begin{tabular}{|c|c|c|}
\hline & {$[\ldots]$ emesalli $[. .]$.} & ${ }^{3}[\ldots]$ emesallu-saline solution [...] \\
\hline 3' & NW 3' $\quad[\ldots \ldots .$. & 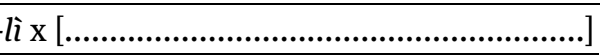 \\
\hline
\end{tabular}

\begin{tabular}{|c|c|c|}
\hline 4' & $\begin{array}{l}{[\ldots \text {.. dāmu?] lā paris dāmu ša ina }} \\
\text { niksišsu așû ...[...] } \\
{[\ldots] \text { teleqqe? dām bīni ina himēti }[. . .]}\end{array}$ & $\begin{array}{l}{ }^{4}[. . . \text { the blood'] is not stopped, the } \\
\text { blood which comes out from its } \\
\text { incision }[. . .]^{5} \text { you take }[. . .][(\text { and }) \text { you } \\
\text {...] tamarisk 'blood' (i.e. resin) in ghee } \\
{[\ldots]}\end{array}$ \\
\hline & \multicolumn{2}{|c|}{ [............. MÚ]D? NU KUD-is MÚD ša ina KUD-šú È x [............................] } \\
\hline & \multicolumn{2}{|c|}{ 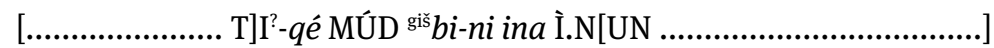 } \\
\hline
\end{tabular}

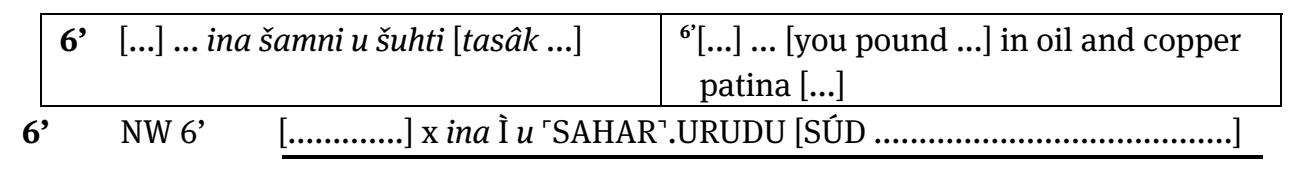

\section{§ IV.11 NZb (AMT 17/2)}

Manuscript: $\quad$ NZb, collated.

Museum Number: $\quad$ Rm 402.

Origin and date: Nineveh, 7th century BC.

Type of tablet: This small fragment represents the left side of a tablet (one or two column).

Measurements: ca. 2,3×4,6×3 cm.

Copy: $\quad$ AMT 17/2.

Photo/CDLI nr.: $\quad$ Plate 36/P424742.

Literature: $\quad$ Borger 1975: 280.

\section{Edition}

\begin{tabular}{|c|c|c|}
\hline 1' & Gula bullițīma [...] & ${ }^{1}$ Gula keep (me) healthy [...] \\
\hline & NZb 1' $\quad{ }^{r d} G u-l a$ & .................................... \\
\hline
\end{tabular}




\begin{tabular}{|ll|l|}
\hline 2' & šalāšat ka'inimma [...] & 2'Three invocations [for ...] \\
\hline 2' & NZb 2' & 3 KA.INIM.MA [................................................................................. \\
\hline
\end{tabular}

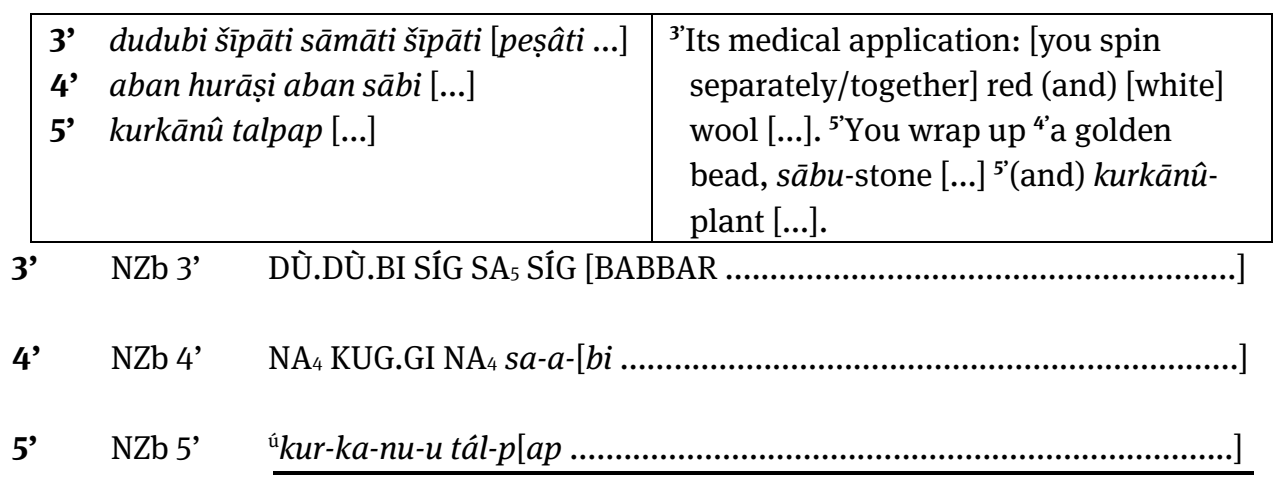

\begin{tabular}{|c|c|c|c|}
\hline $\begin{array}{l}\text { 6' } \\
7 \\
8^{\prime} \\
9^{\prime} \\
10^{\prime} \\
11 \\
12 \\
\text { ', }\end{array}$ & \multicolumn{2}{|c|}{$\begin{array}{l}\text { šiptu abkū ìnāšu [...] } \\
\text { ēmuršuma [...] } \\
\text { ana ețemmi nāq mê }[\text { inaqqi? ....] } \\
\text { ai ițîka }[. . .] \\
\text { ai ițhîka [...] } \\
\text { ina šèrti ina napāhi }[. . .]\end{array}$} & 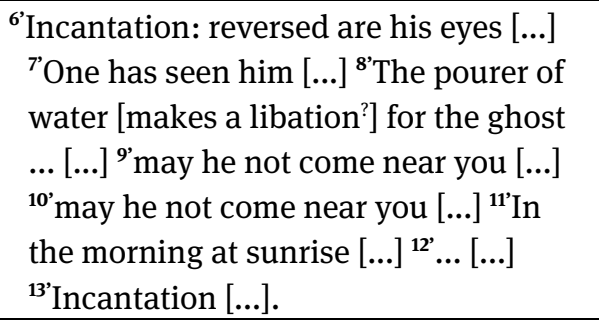 \\
\hline 6' & NZb 6' & \multicolumn{2}{|c|}{ 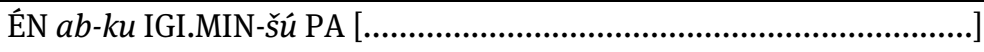 } \\
\hline 7’ & NZb 7’ & \multicolumn{2}{|c|}{ 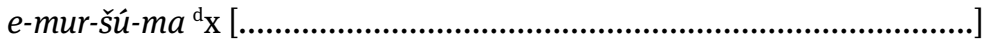 } \\
\hline 8' & NZb 8' & \multicolumn{2}{|c|}{ 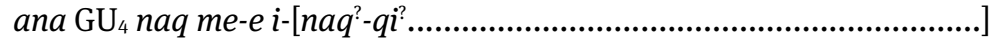 } \\
\hline $9^{\prime}$ & NZb 9' & \multicolumn{2}{|c|}{ 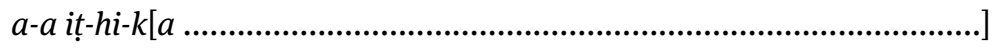 } \\
\hline 10 ' & NZb 10’ & \multicolumn{2}{|c|}{ 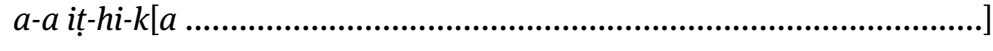 } \\
\hline l1' & NZb 11' & \multicolumn{2}{|c|}{ 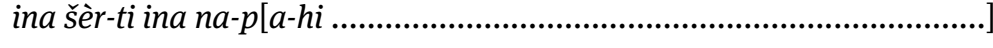 } \\
\hline 12 & NZb 12' & \multicolumn{2}{|c|}{ na-ás-x x x $[\ldots \ldots \ldots \ldots \ldots \ldots$} \\
\hline 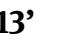 & NZb 13' & \multicolumn{2}{|c|}{ ÉN x } \\
\hline
\end{tabular}

